\title{
MULTIPLIERS ON A NEW CLASS OF BANACH ALGEBRAS, LOCALLY COMPACT QUANTUM GROUPS, AND TOPOLOGICAL CENTRES
}

\author{
ZHIGUO HU, MATTHIAS NEUFANG, AND ZHONG-JIN RUAN
}

\begin{abstract}
We study multiplier algebras for a large class of Banach algebras which contains the group algebra $L_{1}(G)$, the Beurling algebras $L_{1}(G, \omega)$, and the Fourier algebra $A(G)$ of a locally compact group $G$. This study yields numerous new results and unifies some existing theorems on $L_{1}(G)$ and $A(G)$ through an abstract Banach algebraic approach. Applications are obtained on representations of multipliers over locally compact quantum groups and on topological centre problems. In particular, five open problems in abstract harmonic analysis are solved.
\end{abstract}

\section{InTRODUCTION}

Let $A$ be a Banach algebra. Canonically associated with $A$ are the Banach algebras $L M(A)$ and $R M(A)$ of left, respectively right, multipliers on $A$. The main theme of the present paper is to study these algebras for a large class of Banach algebras that includes the most prominent objects of interest in abstract harmonic analysis such as the group algebra $L_{1}(G)$, and, more generally, the weighted convolution (Beurling) algebras $L_{1}(G, \omega)$, as well as the Fourier algebra $A(G)$ of a locally compact group $G$. We shall investigate the multiplier algebras over $A$ from very different aspects as outlined below, which will lead to various intriguing applications. Before discussing the latter in detail, let us briefly emphasize the main virtues of our approach and contributions.

- We bring together a wide spectrum of areas in functional analysis, ranging from general Banach algebra theory and abstract harmonic analysis over locally compact quantum groups to topological centre problems.

- Our approach is Banach algebraic in nature, and yields completely new results on the one hand, while on the other hand unifying existing theorems. In the latter case, despite the much greater generality of our results, the proofs we present are often even quicker than the original ones.

2000 Mathematics Subject Classification. Primary: 43A10, 43A20, 43A30, 46H05.

Key words and phrases. Banach algebras, multipliers, locally compact groups and quantum groups, topological centres.

The first and the second authors were partially supported by NSERC. The third author was partially supported by the National Science Foundation DMS-0500535. 
- As samples of our applications, we answer five open problems from the literature: three from Lau-Ülger [27] (namely, questions 6f), 6h), and 6i)), one from DalesLau [4], and one from Neufang-Ruan-Spronk [37]. In fact, our abstract approach as mentioned above enables us to answer two of those questions - [27, question $6 \mathrm{~h})]$ and the problem raised in [4, Example 4.3] - even in a stronger form than they were originally conjectured.

We shall now describe our results more precisely, grouped into four major topics.

- Characterizing the algebra $A$ inside its multiplier algebras.

- Representation theory for multipliers over locally compact quantum groups.

- Characterizing the algebra $A$ inside its bidual $A^{* *}$ via multipliers.

- Multipliers and topological centres.

The paper is organized as follows. In Section 2, we fix our notation, give definitions, and recall preliminary results on multipliers on Banach algebras and topological centres of biduals of Banach algebras. We provide some background on the theory of locally compact quantum groups, and give a quick proof of the fact that the predual of the latter always carries a faithful completely contractive Banach algebra structure (which is well-known for Kac algebras). We further prove that the predual of any co-amenable locally compact quantum group has an approximate identity consisting of normal states. This interesting result is of potential significance in extending results on locally compact groups to locally compact quantum groups.

In Section 3, we introduce the class of Banach algebras of type $(M)$. Roughly speaking, a Banach algebra $A$ is of type $(M)$ if an algebraic form of Kakutani-Kodaira theorem on locally compact groups holds for $A$. In fact, consideration of this class of Banach algebras is motivated by some Kac algebraic structure results on $L_{\infty}(G)$ and $V N(G)$ of Kakutani-Kodaira flavour (see Remark 7(a)). For a Banach algebra $A$ of type $(M)$, it is shown that $A$ can be characterized inside its multiplier algebras in terms of the behaviour of multipliers of $A$ on $A^{*}$ and $A^{* *}$, respectively.

For a locally compact group $G$, Neufang, Ruan and Spronk proved in [37] a representation theorem for the measure algebra $M(G)$ and the completely bounded multiplier algebra $M_{c b} A(G)$ on $B\left(L_{2}(G)\right)$. In Section 4, the group algebra $L_{1}(G)$, the Beurling algebras $L_{1}(G, \omega)$, and the Fourier algebra $A(G)$ of an amenable group $G$ are shown to be of type $(M)$. Consequently, we obtain two dual characterizations on the images of $L_{1}(G)$ and $A(G)$ under the representations of Neufang-Ruan-Spronk, which answer an open question raised in [37, Remark 4.9]. At the end of Section 4, we present such a characterization for more general locally compact quantum groups under the representation 
proved recently by Junge-Neufang-Ruan [21]. A new characterization on amenability of $G$ is also obtained in this section.

Section 5 is inspired by the pioneering work [27] of Lau-Ülger. It starts with an abstract form of [27, Theorem 5.4] on $L_{1}(G)$ for Banach algebras of type $(M)$ (Theorem 18). This result also includes a dual version of [27, Theorem 5.4] so that the open question $6 \mathrm{~h})$ in $[27]$ on $A(G)$ is answered here in its original form. It clarifies the concern raised

in Dales-Lau [4, Example 4.3] regarding the validity of [27, Theorem 5.4] for general $G$ (see Remark 20), and provides a unified approach to $L_{1}(G)$ and $A(G)$ in a more general Banach algebra framework. Additional results on multipliers are presented in this section, and they are applied to improve Theorem 18 for a subclass of Banach algebras of type $(M)$ (Corollary 28). Furthermore, a result on the Fourier-Stieltjes algebra $B(G)$, dual to Ghahramani-Lau-Losert [10, Proposition 2.4(ii)] on $M(G)$, is obtained (Theorem 29), which helps to enhance Theorem 18 further for $L_{1}(G)$ and $A(G)$. In particular, it is shown that both Lau-Ülger [27, Theorem 5.4] and the more recent [31, Theorem 3.2] by Miao can be strengthened (Corollary 30). We also prove results on topological centres of $A^{* *}$, which closely mingle with results on multipliers and characterizations of $A$ inside $A^{* *}$. Throughout this part, multipliers play a crucial role in characterizing $A$ inside $A^{* *}$ and topological centre problems. The section ends with characterizations of the left/right strong Arens irregularity of Banach algebras of type $(M)$ (Theorem 34).

The paper concludes with Section 6, which contains some consequences of earlier results in the paper and the counterexample $S U(3)$ by Losert, who showed that $A(S U(3))$ is not strongly Arens irregular. In particular, two more open questions in Lau-Ülger [27] are answered in this section.

\section{Preliminaries}

Throughout this paper, $A$ denotes a Banach algebra and $\left\langle A^{*} A\right\rangle$ (resp. $\left.\left\langle A A^{*}\right\rangle\right)$ denotes the closed linear span of $A^{*} A$ (resp. $A A^{*}$ ) in $A^{*}$. Then it is known from Cohen's factorization theorem that $\left\langle A^{*} A\right\rangle=A^{*} A$ and $\left\langle A A^{*}\right\rangle=A A^{*}$ if $A$ has a bounded approximate identity (BAI). If $A$ is separable with a BAI $\left(e_{\alpha}\right)$, then a sequential BAI of $A$ can be constructed inductively from $\left(e_{\alpha}\right)$ and a countable dense subset of $A$. See Ülger [46, Lemma 3.2] for a more general discussion of such a fact.

$A$ is called weakly sequentially complete (WSC) if every weakly Cauchy sequence in $A$ is weakly convergent. It is well-known that the predual of any von Neumann algebra is WSC (cf. Takesaki [44, Corollary III.5.2]). 
Let $B(A)$ be the Banach algebra of bounded linear operators on $A$ and let $L M(A)$ (resp. $R M(A))$ be the left (resp. right) multiplier algebra of $A$. That is,

$$
\begin{aligned}
& L M(A)=\{T \in B(A): T(a b)=T(a) b \text { for all } a, b \in A\}, \\
& R M(A)=\{T \in B(A): T(a b)=a T(b) \text { for all } a, b \in A\} .
\end{aligned}
$$

As norm closed subalgebras of $B(A)$ and $B(A)^{o p}$ (the opposite algebra of $B(A)$ ), respectively, $L M(A)$ and $R M(A)$ are Banach algebras.

For $a \in A, l_{a}$ and $r_{a}$ will denote the linear maps $b \mapsto a b$ and $b \mapsto b a$ on $A$, respectively. Then $l_{a} \in L M(A)$ and $r_{a} \in R M(A)$ with $\left\|l_{a}\right\| \leq\|a\|$ and $\left\|r_{a}\right\| \leq\|a\|$. It is easy to see that $a \mapsto l_{a}$ (resp. $a \mapsto r_{a}$ ) is injective if and only if $A$ is left (resp. right) faithful. In particular, if $A$ has a BAI of bound $k$, then $\left\|l_{a}\right\| \geq k^{-1}\|a\|$ and $\left\|r_{a}\right\| \geq k^{-1}\|a\|$ for all $a \in A$. In this case, $A$ is identified with a norm closed left (resp. right) ideal in $L M(A)$ (resp. $R M(A))$.

Let - and $\triangle$ denote the left and right Arens products on $A^{* *}$, respectively. By definition, the left Arens product on $A^{* *}$ is induced by the left $A$-module structure on $A$. That is, for $m, n \in A^{* *}, f \in A^{*}$, and $a, b \in A$, we have

$$
\langle m \cdot n, f\rangle=\langle m, n \cdot f\rangle
$$

where $\langle n \cdot f, a\rangle=\langle n, f \cdot a\rangle$ and $\langle f \cdot a, b\rangle=\langle f, a b\rangle$. Similarly, the right Arens product on $A^{* *}$ is defined when $A$ is considered as a right $A$-module. It is known that

$$
m \cdot n=\text { weak }^{*}-\lim _{\alpha} \lim _{\beta} a_{\alpha} b_{\beta} \text { and } m \triangle n=\text { weak }^{*}-\lim _{\beta} \lim _{\alpha} a_{\alpha} b_{\beta}
$$

whenever $\left(a_{\alpha}\right)$ and $\left(b_{\beta}\right)$ are nets in $A$ such that $a_{\alpha} \longrightarrow m$ and $b_{\beta} \longrightarrow n$ in the weak*topology on $A^{* *}$.

$A$ is said to be Arens regular if . and $\triangle$ coincide on $A^{* *}$. The left and the right topological centres of $A^{* *}$ are defined, respectively, as

$$
Z_{t}^{(l)}\left(A^{* *}\right)=\left\{m \in A^{* *}: \text { the map } n \mapsto m \cdot n \text { is weak } \text {-weak }^{*} \text { continuous on } A^{* *}\right\}
$$

and

$$
Z_{t}^{(r)}\left(A^{* *}\right)=\left\{m \in A^{* *}: \text { the map } n \mapsto n \triangle m \text { is weak*-weak }{ }^{*} \text { continuous on } A^{* *}\right\}
$$

It is easy to see that $Z_{t}^{(l)}\left(A^{* *}\right)=\left\{m \in A^{* *}: m \cdot n=m \triangle n\right.$ for all $\left.n \in A^{* *}\right\}$, and $Z_{t}^{(r)}\left(A^{* *}\right)=\left\{m \in A^{* *}: n \triangle m=n \cdot m\right.$ for all $\left.n \in A^{* *}\right\}$. Therefore, $A$ is Arens regular if and only if $Z_{t}^{(l)}\left(A^{* *}\right)=A^{* *}=Z_{t}^{(r)}\left(A^{* *}\right)$. If $A$ is a commutative Banach algebra, then $Z_{t}^{(l)}\left(A^{* *}\right)=Z_{t}^{(r)}\left(A^{* *}\right)$ is just the algebraic centre $Z\left(A^{* *}\right)$ of $A^{* *}$ (equipped with either of Arens products). Readers are referred to Dales [3], Dales-Lau [4], and Palmer [38] for more information on Arens products and topological centres. 
For $m \in A^{* *}$, let $\lambda_{m}, \lambda^{m}, \rho_{m}$, and $\rho^{m}$ be the maps $n \mapsto m \cdot n, n \mapsto m \triangle n, n \mapsto n \cdot m$, and $n \mapsto n \triangle m$ on $A^{* *}$, respectively. Then for all $a \in A, \lambda_{\tilde{a}}=\lambda^{\tilde{a}}=\left(l_{a}\right)^{* *}$ and $\rho_{\tilde{a}}=\rho^{\tilde{a}}=\left(r_{a}\right)^{* *}$, where $a \mapsto \tilde{a}$ is the canonical embedding $A \hookrightarrow A^{* *}$. The above descriptions of $Z_{t}^{(l)}\left(A^{* *}\right)$ and $Z_{t}^{(r)}\left(A^{* *}\right)$ imply that

$$
m \in Z_{t}^{(l)}\left(A^{* *}\right) \text { if and only if } \lambda_{m}=\lambda^{m},
$$

and

$$
m \in Z_{t}^{(r)}\left(A^{* *}\right) \text { if and only if } \rho_{m}=\rho^{m} .
$$

In this paper, we are mainly interested in the case when $A$ is the group algebra $L_{1}(G)$, the Fourier algebra $A(G)$, or more generally, the predual $M_{*}$ of a locally compact quantum group $\mathbb{G}=(M, \Gamma, \varphi, \psi)$ introduced by Kustermans and Vaes (see [24] and [48]), where $(M, \Gamma)$ is a Hopf-von Neumann algebra, $\varphi$ is a normal faithful left invariant weight on $(M, \Gamma)$, and $\psi$ is a normal faithful right invariant weight on $(M, \Gamma)$. In particular, every Kac algebra is a locally compact quantum group. For details on Kac algebras, readers are referred to Enock-Schwartz's book [8].

It is known by Kustermans and Vaes that every locally compact quantum group $\mathbb{G}$ has a dual locally compact quantum group $\widehat{\mathbb{G}}=(\widehat{M}, \widehat{\Gamma}, \widehat{\varphi}, \widehat{\psi})$. If $\mathbb{G}$ is a Kac algebra, then so is $\widehat{\mathbb{G}}$. The Pontryagin duality theorem for locally compact abelian groups extends to locally compact quantum groups. That is, for all locally compact quantum groups $\mathbb{G}$, we have $\widehat{\widehat{\mathbb{G}}}=\mathbb{G}$.

Let $G$ be a locally compact group. Define $\Gamma_{a}: L_{\infty}(G) \longrightarrow L_{\infty}(G) \bar{\otimes} L_{\infty}(G)(=$ $\left.L_{\infty}(G \times G)\right)$ by

$$
\left(\Gamma_{a} f\right)(x, y)=f(x y) \quad\left(f \in L_{\infty}(G), x, y \in G\right),
$$

where $\bar{\otimes}$ denotes the von Neumann algebra tensor product. Then $\left(L_{\infty}(G), \Gamma_{a}\right)$ is a commutative Hopf-von Neumann algebra. If we let $\varphi$ and $\psi$ be a left and a right Haar integral on $G$, respectively, then $\mathbb{G}_{a}=\left(L_{\infty}(G), \Gamma_{a}, \varphi, \psi\right)$ is a commutative locally compact quantum group.

Let $\lambda$ be the left regular representation of $G$, and $V N(G)$ the von Neumann algebra generated by $\lambda$. Then $\lambda(x) \mapsto \lambda(x) \otimes \lambda(x)(x \in G)$ determines a co-multiplication $\widehat{\Gamma_{a}}: V N(G) \longrightarrow V N(G) \bar{\otimes} V N(G)$. Therefore, $\left(V N(G), \widehat{\Gamma_{a}}\right)$ is a Hopf-von Neumann algebra. Furthermore, if we let $\widehat{\varphi}$ be the Plancherel weight on $V N(G)$ (cf. Takesaki [45, Definition VII.3.2]), and let $\widehat{\psi}=\widehat{\varphi}$, then $\mathbb{G}_{s}=\left(V N(G), \widehat{\Gamma_{a}}, \widehat{\varphi}, \widehat{\psi}\right)$ is also a locally compact quantum group. It is well-known that both $\mathbb{G}_{a}$ and $\mathbb{G}_{s}$ are Kac algebras, and $\widehat{\mathbb{G}_{a}}=\mathbb{G}_{s}$.

Let $\mathbb{G}=(M, \Gamma, \varphi, \psi)$ be a locally compact quantum group. As the predual of the von Neumann algebra $M, M_{*}$ has a canonical operator space structure. Readers are referred 
to the recent books by Effros-Ruan [7], Paulsen [39], and Pisier [40], respectively, for detailed information on operator space theory.

Since the co-multiplication $\Gamma$ is a normal isometric unital *-homomorphism from $M$ into $M \bar{\otimes} M$, it is well-known that its pre-adjoint $\Gamma_{*}: M_{*} \widehat{\otimes} M_{*} \rightarrow M_{*}$ induces an associative completely contractive multiplication on $M_{*}$, denoted by $\star$, where $\widehat{\otimes}$ is the operator space projective tensor product (cf. Ruan $[41,42]$ ). For $\mathbb{G}=\mathbb{G}_{a}, \star$ is just the usual convolution on $L_{1}(G)$, and for $\mathbb{G}=\mathbb{G}_{s}, \star$ gives the pointwise multiplication on $A(G)$.

Kraus and Ruan showed in [22, Proposition 4.1] that $M_{*}$ with the multiplication $\star$ is a faithful Banach algebra if $\mathbb{G}$ is a Kac algebra. This is still true for a general locally compact quantum group following essentially the same arguments of [22].

Proposition 1. Let $\mathbb{G}=(M, \Gamma, \varphi, \psi)$ be a locally compact quantum group. Then $M_{*}$ is a faithful completely contractive Banach algebra.

Proof. Assume $\omega_{0} \in M_{*}$ and $\omega \star \omega_{0}=0$ for all $\omega \in M_{*}$. Then we have

$$
\left\langle\Gamma(x)(y \otimes 1), \omega \otimes \omega_{0}\right\rangle=\left\langle\Gamma(x),(y \cdot \omega) \otimes \omega_{0}\right\rangle=\left\langle x,(y \cdot \omega) \star \omega_{0}\right\rangle=0
$$

for all $x, y \in M$ and $\omega \in M_{*}$, where $y \cdot \omega \in M_{*}$ is given by $\langle x, y \cdot \omega\rangle=\langle x y, \omega\rangle$. Since $\Gamma(M)(M \otimes 1)$ is weak* dense in $M \bar{\otimes} M$ (cf. Van Daele [48]), we conclude that $\omega_{0}=0$.

Similarly, we can prove that $\omega_{0} \star \omega=0$ for all $\omega \in M_{*}$ implies $\omega_{0}=0$, since $\Gamma(M)(1 \otimes$ $M)$ is also weak* dense in $M \bar{\otimes} M$. Therefore, $M_{*}$ with the multiplication $\star$ is a faithful Banach algebra.

For a locally compact quantum group $\mathbb{G}=(M, \Gamma, \varphi, \psi)$, the Banach algebra $M_{*}$ with the multiplication $\star$ will be denoted by $L_{1}(\mathbb{G})$. Accordingly, $M$ will be denoted by $L_{\infty}(\mathbb{G})$. Let $L_{2}(M, \varphi)$ (resp. $L_{2}(M, \psi)$ ) be the Hilbert space obtained from the GNSconstruction for $\varphi$ (resp. $\psi$ ). One can show that $L_{2}(M, \varphi) \cong L_{2}(M, \psi)$. We will denote this Hilbert space by $L_{2}(\mathbb{G})$. It is also known that $L_{2}(\mathbb{G}) \cong L_{2}(\widehat{\mathbb{G}})$.

Every locally compact quantum group $\mathbb{G}$ has a canonical co-involution $R$ (the "unitary antipode"). That is, $R: M \longrightarrow M$ is a ${ }^{*}$-anti-homomorphism satisfying $R^{2}=i d$ and

$$
\Gamma \circ R=\sigma(R \otimes R) \circ \Gamma
$$

where $\sigma$ is the flip map on $L_{2}(\mathbb{G}) \otimes L_{2}(\mathbb{G})$ (cf. Kustermans-Vaes [24] and Van Daele [48]). Then $R$ induces an involution on $L_{1}(\mathbb{G})$ given by

$$
\left\langle x, f^{o}\right\rangle=\overline{\left\langle f, R\left(x^{*}\right)\right\rangle}\left(f \in L_{1}(\mathbb{G}), x \in L_{\infty}(\mathbb{G})\right),
$$

so that $L_{1}(\mathbb{G})$ becomes an involutive Banach algebra.

$\mathbb{G}$ is called co-amenable if $L_{1}(\mathbb{G})$ has a BAI. Bédos-Tuset [2, Theorem 3.1] shows that $\mathbb{G}$ is co-amenable if and only if $L_{1}(\mathbb{G})$ has a contractive left (resp. right) approximate 
identity. For a locally compact group $G, L_{1}(G)$ always has a BAI, and $A(G)$ has a BAI precisely when $G$ is amenable (cf. Leptin [28]). Therefore, for all locally compact groups $G, \mathbb{G}_{a}$ is co-amenable, and $\mathbb{G}_{s}$ is co-amenable if and only if $G$ is amenable. We note that in these two classical cases, $\mathbb{G}$ is co-amenable if and only if $L_{1}(\mathbb{G})$ has a BAI consisting of normal states on $L_{\infty}(\mathbb{G})$. We show below that this assertion holds for all locally compact quantum groups.

Theorem 2. Let $\mathbb{G}=(M, \Gamma, \varphi, \psi)$ be a locally compact quantum group. Then $\mathbb{G}$ is co-amenable if and only if $L_{1}(\mathbb{G})$ has a BAI consisting of normal states on $L_{\infty}(\mathbb{G})$.

Proof. Given a locally compact quantum group $\mathbb{G}$, there exists a left fundamental unitary operator $W$ defined on $L_{2}(\mathbb{G}) \otimes L_{2}(\mathbb{G})$ satisfying

$$
\Gamma(x)=W^{*}(1 \otimes x) W
$$

for all $x \in M$. We let $A_{\mathbb{G}}$ denote the $\mathrm{C}^{*}$-subalgebra of $M$ generated by $\{(\iota \otimes \omega) W: \omega \in$ $\left.B\left(L_{2}(\mathbb{G})\right)_{*}\right\}$. It is known from Bédos-Tuset $[2, \mathrm{p} .872-873]$ that $\mathbb{G}$ is co-amenable if and only if there exists a state $\varepsilon$ on $A_{\mathbb{G}}$ such that

$$
(\varepsilon \otimes \iota) \Gamma=\iota \text { and }(\iota \otimes \varepsilon) \Gamma=\iota,
$$

i.e., $\varepsilon$ is a unital element in the Banach algebra $\left(A_{\mathbb{G}}\right)^{*}$. In this case, we have

$$
\varepsilon \circ R=\varepsilon \text { and }(\varepsilon \otimes \iota)(W)=1,
$$

where $R$ denotes the unitary antipode of $\mathbb{G}$ on $A_{\mathbb{G}}$ (respectively, on $M$ ) and the operator $(\varepsilon \otimes \iota)(W) \in B\left(L_{2}(\mathbb{G})\right)$ is determined by

$$
\langle(\varepsilon \otimes \iota)(W), \omega\rangle=\langle\varepsilon,(\iota \otimes \omega)(W)\rangle
$$

for all $\omega \in B\left(L_{2}(\mathbb{G})\right)_{*}$. We have $R^{2}=i d_{M}$, and $\omega^{o}=\omega^{*} \circ R$ induces a Banach algebra involution on $L_{1}(\mathbb{G})$ (where $\left.\omega^{*}(x)=\overline{\omega\left(x^{*}\right)}\right)$.

Assume now that $\mathbb{G}$ is co-amenable. Let $\tilde{\varepsilon}$ be a state extension of $\varepsilon$ to $M$. Then it is easy to see that

$$
(\tilde{\varepsilon} \otimes \iota)(W)=(\varepsilon \otimes \iota)(W)=1 .
$$

We can also conclude that

$$
(\tilde{\varepsilon} \circ R \otimes \iota)(W)=(\varepsilon \circ R \otimes \iota)(W)=1 .
$$

However, it is not necessarily true that $\tilde{\varepsilon} \circ R=\tilde{\varepsilon}$ on $M$ (since $\tilde{\epsilon}$ is not normal on $M$ ). But we can replace $\tilde{\varepsilon}$ by

$$
\tilde{\tilde{\varepsilon}}=\frac{\tilde{\varepsilon}+\tilde{\varepsilon} \circ R}{2}=\frac{\tilde{\varepsilon}+\tilde{\varepsilon}^{o}}{2}
$$

and thus can obtain a state extension $\tilde{\tilde{\varepsilon}}$ of $\varepsilon$ to $M$ satisfying (1). 
Since $\tilde{\tilde{\varepsilon}} \in M^{*}=L_{1}(\mathbb{G})^{* *}$, there exists a net $\left(\omega_{i}\right)$ of normal states on $M$ such that $\omega_{i} \rightarrow \tilde{\tilde{\varepsilon}}$ in the $\sigma\left(M^{*}, M\right)$ topology. Moreover, since $M$ is standardly represented on $L_{2}(\mathbb{G})$, there exist unit vectors $u_{i}$ in $L_{2}(\mathbb{G})$ such that $\omega_{i}=\omega_{u_{i}}$ for all $i$. Using the same argument as that given in the proof of [2, Theorem 3.1], we can get that, for every $v \in L_{2}(\mathbb{G})$,

$$
\begin{aligned}
\lim _{i}\left\langle W\left(u_{i} \otimes v\right) \mid\left(u_{i} \otimes v\right)\right\rangle & =\lim _{i}\left\langle\left(\iota \otimes \omega_{v}\right)(W) u_{i} \mid u_{i}\right\rangle=\left\langle\tilde{\tilde{\varepsilon}},\left(\iota \otimes \omega_{v}\right)(W)\right\rangle \\
& =\left\langle(\tilde{\tilde{\varepsilon}} \otimes \iota)(W), \omega_{v}\right\rangle=\omega_{v}(1)=\langle v \mid v\rangle \\
& =\lim _{i}\left\langle u_{i} \otimes v \mid u_{i} \otimes v\right\rangle .
\end{aligned}
$$

Then we have

$\left\|W\left(u_{i} \otimes v\right)-\left(u_{i} \otimes v\right)\right\|^{2}=\left\|W\left(u_{i} \otimes v\right)\right\|^{2}+\left\|\left(u_{i} \otimes v\right)\right\|^{2}-2 R e\left\langle W\left(u_{i} \otimes v\right) \mid\left(u_{i} \otimes v\right)\right\rangle \rightarrow 0$.

Therefore, for all $x \in M$,

$$
\left|\omega_{i} \star \omega_{v}(x)-\omega_{v}(x)\right|=\left|\left\langle W^{*}(1 \otimes x) W\left(u_{i} \otimes v\right)-(1 \otimes x)\left(u_{i} \otimes v\right) \mid u_{i} \otimes v\right\rangle\right| \rightarrow 0 .
$$

This shows that $\left(\omega_{i}\right)$ is a contractive "weak left approximate identity" of $L_{1}(\mathbb{G})$.

Since $\omega_{i}^{o}=\omega_{i}^{*} \circ R=\omega_{i} \circ R \rightarrow \tilde{\tilde{\varepsilon}} \circ R=\tilde{\tilde{\varepsilon}}$, replacing $\left(\omega_{i}\right)$ above by $\left(\omega_{i}^{o}\right)$, we can similarly prove that $\left(\omega_{i}^{o}\right)$ is also a contractive "weak left approximate identity" of $L_{1}(\mathbb{G})$.

Now let us consider the net $\left(\left(\omega_{i}, \omega_{i}^{o}\right)\right)_{i}$ in the Banach algebra $L_{1}(\mathbb{G}) \oplus_{1} L_{1}(\mathbb{G})$. Note that $\left(L_{1}(\mathbb{G}) \oplus_{1} L_{1}(\mathbb{G})\right)^{*}=M \oplus_{\infty} M$. From the above discussion, $\left(\left(\omega_{i}, \omega_{i}^{o}\right)\right)_{i}$ is a (bounded) weak left approximate identity of $L_{1}(\mathbb{G}) \oplus_{1} L_{1}(\mathbb{G})$. By the standard convexity argument, we can obtain a left approximate identity of $L_{1}(\mathbb{G}) \oplus_{1} L_{1}(\mathbb{G})$ in the convex hull $\operatorname{co}\left\{\left(\omega_{i}, \omega_{i}^{o}\right)\right\}$ of $\left\{\left(\omega_{i}, \omega_{i}^{o}\right)\right\}$. Thus, this left approximate identity can be written as $\left(\left(\tilde{\omega}_{j}, \tilde{\omega}_{j}^{\prime}\right)\right)_{j}$, where $\tilde{\omega}_{j} \in \operatorname{co}\left\{\left(\omega_{i}\right)\right\}$ and $\tilde{\omega}_{j}^{\prime} \in \operatorname{co}\left\{\left(\omega_{i}^{o}\right)\right\}$ are normal states on $M$, and we actually have $\tilde{\omega}_{j}^{\prime}=\tilde{\omega}_{j}^{o}$.

Therefore, $\left(\tilde{\omega}_{j}\right)$ is also a right approximate identity of $L_{1}(\mathbb{G})$. This shows that $\left(\tilde{\omega}_{j}\right)$ is a BAI of $L_{1}(\mathbb{G})$ consisting of states on $M=L_{\infty}(\mathbb{G})$.

\section{Multipliers on a NeW Class of Banach algebras}

Let $A$ be a Banach algebra. For $\mu \in L M(A)$ (resp. $\mu \in R M(A)$ ), we will write $\mu \in A$ if $\mu=l_{a}$ (resp. $\mu=r_{a}$ ) for some $a \in A$. We start with the following result on how a multiplier on $A$ to be implemented by an element from $A$ is determined by its behaviour on $A^{*}$ and $A^{* *}$, respectively.

Theorem 3. Let $\mu \in L M(A)$ (resp. $\mu \in R M(A)$ ). Consider the following statements.

(i) $\mu \in A$.

(ii) $\mu^{* *}=\lambda_{m}$ (resp. $\left.\mu^{* *}=\rho^{m}\right)$ for some $m \in A^{* *}$. 
(iii) $\mu^{*}\left(A^{*}\right) \subseteq\left\langle A^{*} A\right\rangle$ (resp. $\left.\mu^{*}\left(A^{*}\right) \subseteq\left\langle A A^{*}\right\rangle\right)$.

Then

(a) (i) $\Longrightarrow$ (ii) $\Longrightarrow$ (iii).

(b) (i) $\Longrightarrow$ (ii) $\Longleftrightarrow$ (iii) if $A$ has a BAI.

(c) (i) $\Longleftrightarrow$ (ii) $\Longleftrightarrow$ (iii) if $A$ is WSC with a sequential BAI.

Proof. (a) Obviously, (i) $\Longrightarrow$ (ii).

To prove (ii) $\Longrightarrow$ (iii) for $L M(A)$, suppose $\mu \in L M(A)$ and $\mu^{* *}=\lambda_{m}$ for some $m \in A^{* *}$. Choose a net $\left(a_{\alpha}\right)$ in $A$ such that $a_{\alpha} \longrightarrow m$ in the weak* topology on $A^{* *}$. Let $f \in A^{*}$. Then for all $n_{0} \in\left\langle A^{*} A\right\rangle^{\perp}$, we have

$$
\left\langle n_{0}, \mu^{*}(f)\right\rangle=\left\langle\mu^{* *}\left(n_{0}\right), f\right\rangle=\left\langle m \cdot n_{0}, f\right\rangle=\lim _{\alpha}\left\langle n_{0}, f \cdot a_{\alpha}\right\rangle=0 .
$$

Therefore, $\mu^{*}(f) \in\left\langle A^{*} A\right\rangle$. The proof for the case $\mu \in R M(A)$ is similar.

(b) Assume that $A$ has a BAI $\left(e_{i}\right)$ and $\mu \in L M(A)$. We only need to show that (iii) $\Longrightarrow$ (ii). So, we suppose $\mu^{*}\left(A^{*}\right) \subseteq\left\langle A^{*} A\right\rangle=A^{*} A$. We may assume that $e_{i} \longrightarrow E \in A^{* *}$ in the weak*-topology on $A^{* *}$. It is easy to see that

$$
\mu^{* *}(n)=\mu^{* *}(E) \triangle n \text { and } \mu^{*}(f)=f \triangle \mu^{* *}(E) \text { for all } n \in A^{* *} \text { and } f \in A^{*} .
$$

Let $f \in A^{*}$ and $n \in A^{* *}$. By the assumption, $\mu^{*}(f)=f \triangle \mu^{* *}(E)=g \cdot a$ for some $g \in A^{*}$ and $a \in A$. We have

$$
\left\langle f, \mu^{* *}(E) \triangle n\right\rangle=\left\langle f \triangle \mu^{* *}(E), n\right\rangle=\langle g \cdot a, n\rangle=\langle g, a \cdot n\rangle .
$$

On the other hand, since $\mu^{*}(n \cdot f)=n \cdot \mu^{*}(f)=n \cdot(g \cdot a)$, we have

$$
\left\langle\mu^{* *}(E) \cdot n, f\right\rangle=\left\langle E, \mu^{*}(n \cdot f)\right\rangle=\langle E, n \cdot(g \cdot a)\rangle=\langle a \cdot E \cdot n, g\rangle=\langle a \cdot n, g\rangle .
$$

Therefore, $\mu^{* *}(n)=\mu^{* *}(E) \triangle n=\mu^{* *}(E) \cdot n$ for all $n \in A^{* *}$. That is, $\mu^{* *}=\lambda_{\mu^{* *}(E)}$.

The proof for the case $\mu \in R M(A)$ follows from similar arguments.

(c) Finally, we assume that $A$ is WSC with a sequential BAI $\left(e_{n}\right)$, and $\mu \in \operatorname{LM}(A)$. We prove that (iii) $\Longrightarrow$ (i). The proof for the case $\mu \in R M(A)$ is similar.

From the proof of (b), we see that if $m$ is a weak*-cluster point of $\left(\mu\left(e_{n}\right)\right)$ in $A^{* *}$, then $\mu^{* *}=\lambda_{m}$. Since $\left(A^{* *}, \cdot\right)$ has a right identity, $\left(\mu\left(e_{n}\right)\right)$ has a unique weak*-cluster point and hence it is weak ${ }^{*}$-convergent in $A^{* *}$. Thus, $\left(\mu\left(e_{n}\right)\right)$ is a weakly Cauchy sequence in $A$. By the weakly sequential completeness of $A, \mu\left(e_{n}\right) \longrightarrow a$ weakly for some $a \in A$. For all $b \in A$, we have $\mu\left(e_{n}\right) b=\mu\left(e_{n} b\right) \longrightarrow \mu(b)=a b$. Therefore, $\mu=l_{a}$, i.e., $\mu \in A$.

It is seen from the proof of (iii) $\Longrightarrow$ (i) in (c), that if $A$ is WSC with a sequential approximate identity $\left(e_{n}\right)$ (not necessarily bounded), then we still have $\left[\mu^{*}\left(A^{*}\right) \subseteq\right.$ $\left.A^{*} A\right] \Longrightarrow[\mu \in A]$ (resp. $\left.\left[\mu^{*}\left(A^{*}\right) \subseteq A A^{*}\right] \Longrightarrow[\mu \in A]\right)$. In fact, in this case, $\left(\mu\left(e_{n}\right)\right)$ is weakly Cauchy for all $\mu \in L M(A)$ (resp. $\mu \in R M(A)$ ) satisfying $\mu^{*}\left(A^{*}\right) \subseteq A^{*} A$ 
(resp. $\left.\mu^{*}\left(A^{*}\right) \subseteq A A^{*}\right)$. This kind of arguments, involving WSC Banach algebras with a sequential BAI, is in the spirit of Lau-Ülger [27, Theorem 3.4a)] and Ülger [46, Lemma $3.1]$.

We note that in the proof of (c), it is crucial that the cardinality of a BAI of $A$ is dominated by a cardinal level of the weak completeness of $A$. It turns out that for a general Banach algebra $A$, it may be very difficult to obtain a cardinal level of the weak completeness of $A$ higher than $\aleph_{0}$.

On the other hand, it happens that a Banach algebra $A$ can have a BAI without any sequential BAI. For example, for a locally compact group $G, L_{1}(G)$ has a sequential BAI if and only if $G$ is metrizable, and $A(G)$ has a sequential BAI if and only if $G$ is amenable and $\sigma$-compact (cf. Remark 15).

To deal with the general situation where the size of a BAI is not controlled by a cardinal level of the weak completeness of $A$, we will focus on Banach algebras possessing "large" family of "small" subalgebras. More precisely, we consider those Banach algebras $A$ which have a family $\left\{A_{i}\right\}$ of subalgebras such that each $A_{i}$ has a sequential BAI, and the family $\left\{A_{i}\right\}$ is large enough so that a multiplier on $A$ being in $A$ or not is determined in a certain sense by its behaviour on these subalgebras. The theorem below is a result along this line of approach.

We first note that if $A$ is a Banach algebra with a left (resp. right) approximate identity and $J$ is a closed left (resp. right) ideal in $A$, then for all $\mu \in L M(A)$ (resp. $\mu \in R M(A)),\left.\mu\right|_{J} \in L M(J)$ (resp. $\left.\mu\right|_{J} \in R M(J)$ ).

Theorem 4. Let $A$ be a Banach algebra with a BAI. Assume that there exists a family $\left\{A_{i}\right\}_{i \in \Lambda}$ of closed left (resp. right) ideals in $A$ with the following properties.

(I) Each $A_{i}$ is WSC with a sequential approximate identity.

(II) For each $i \in \Lambda$, there exists a right (resp. left) $A_{i}$-module projection $p_{i}$ from $A$ onto $A_{i}$.

(III) For any $\nu \in L M(A)$ (resp. $\nu \in R M(A)$ ), if $\left.\nu\right|_{A_{i}} \in A_{i}$ for all $i \in \Lambda$, then $\nu \in A$. Then the assertions (i)-(iii) in Theorem 3 are equivalent for all $\mu \in L M(A)$ (resp. $\mu \in R M(A))$.

Proof. Let $\mu \in L M(A)$. By Theorem 3, we only have to prove (iii) $\Longrightarrow$ (i). So, we assume that $\mu^{*}\left(A^{*}\right) \subseteq\left\langle A^{*} A\right\rangle=A^{*} A$.

Fix an $i \in \Lambda$ and let $\mu_{i}=\left.\mu\right|_{A_{i}}$. Note that $\mu_{i} \in L M\left(A_{i}\right)$.

We claim that $\mu_{i}^{*}\left(A_{i}^{*}\right) \subseteq A_{i}^{*} A_{i}$. To see this, let $h \in A_{i}^{*}$ and let $\tilde{h} \in A^{*}$ be any HahnBanach extension of $h$. Then $\mu^{*}(\tilde{h})=f \cdot a$ for some $f \in A^{*}$ and $a \in A$. Note that for all 
$b \in A_{i}, a b=p_{i}(a b)=p_{i}(a) b$, and thus, we have

$$
\left\langle\mu_{i}^{*}(h), b\right\rangle=\langle\tilde{h}, \mu(b)\rangle=\left\langle\mu^{*}(\tilde{h}), b\right\rangle=\langle f, a b\rangle=\left\langle f, p_{i}(a) b\right\rangle=\left\langle\left(\left.f\right|_{A_{i}}\right) \cdot p_{i}(a), b\right\rangle .
$$

Therefore, $\mu_{i}^{*}(h)=\left(\left.f\right|_{A_{i}}\right) \cdot p_{i}(a) \in A_{i}^{*} A_{i}$.

By condition (I) and Theorem 3(c) together with the remark immediately following the proof of Theorem 3, we have $\mu_{i} \in A_{i}$. Since $i \in \Lambda$ is arbitrary, we have $\mu \in A$ from condition (III).

The proof for the case $\mu \in R M(A)$ is similar.

The proof of Theorem 4 shows that the following more general theorem holds.

Theorem 5. Let $A$ be a Banach algebra with a BAI. Assume that for any $\mu$ in $L M(A)$ (resp. $R M(A)$ ), $A$ has a closed subalgebra $B$ of the type as in Theorem 4 such that

(1) for each $f \in A^{*} A$ (resp. $\left.f \in A A^{*}\right),\left.f\right|_{B} \in B^{*} B$ (resp. $\left.f\right|_{B} \in B B^{*}$ );

(2) $\mu(B) \subseteq B$, and $\mu \in A$ if $\left.\mu\right|_{B} \in B$.

Then the assertions (i)-(iii) in Theorem 3 are equivalent for all $\mu \in L M(A)$ (resp. $\mu \in R M(A))$.

We are thus led to introducing the following concept.

Definition 6. Let $A$ be a Banach algebra as in Theorem 5. Then $A$ is said to be of type $(L M)$ and of type $(R M)$, respectively.

If $A$ is both of type ( $L M)$ and of type $(R M), A$ is said to be of type $(M)$.

Remark 7. (a) Of course, any unital WSC Banach algebra is of type $(M)$. So is a WSC Banach algebra with a sequential BAI. In particular, if $\mathbb{G}$ is a co-amenable locally compact quantum group with $L_{1}(\mathbb{G})$ separable, then $L_{1}(\mathbb{G})$ is of type $(M)$.

We show in the next section that, for all locally compact groups $G, L_{1}(G)$ is of type $(M)$, and it is the case for $A(G)$ when $G$ is amenable. These assertions are proved by using some Kac algebraic structure results on $L_{\infty}(G)$ and $V N(G)$ obtained by $\mathrm{Hu}[17,18]$ and by Hu-Neufang [19]. It is known that the predual of any Hopf-von Neumann algebra is a WSC completely contractive Banach algebra. If those structure results on $L_{\infty}(G)$ and $V N(G)$ would hold for a general locally compact quantum group, then the predual of any co-amenable locally compact quantum group would be of type $(M)$.

(b) We point out that if $A$ is a left ideal in $A^{* *}$ with either a BAI or a sequential bounded right approximate identity, then one can obtain (iii) $\Longrightarrow$ (i) by applying BakerLau-Pym [1, Theorem 3.1], where $A$ should be assumed to be a left (instead of a right) ideal in $A^{* *}$. 


\section{Completely isometric Representations of $L_{1}(G)$ And $A(G)$}

Let $G$ be a locally compact group. Let $\Theta_{r}$ and $\widehat{\Theta}$ be the completely isometric representations of $M(G)$ and $M_{c b} A(G)$ in $C B^{\sigma}\left(B\left(L_{2}(G)\right)\right)$, respectively, where $C B^{\sigma}\left(B\left(L_{2}(G)\right)\right)$ is the space of weak*-weak* continuous completely bounded linear maps on $B\left(L_{2}(G)\right)$ (see Neufang [32] and Neufang-Ruan-Spronk [37]). It is proved in [32] and [37], respectively, that

$$
\Theta_{r}(M(G))=C B_{V N(G)}^{\sigma, L_{\infty}(G)}\left(B\left(L_{2}(G)\right)\right)
$$

and

$$
\widehat{\Theta}\left(M_{c b} A(G)\right)=C B_{L_{\infty}(G)}^{\sigma, V N(G)}\left(B\left(L_{2}(G)\right)\right),
$$

where, for subalgebras $M$ and $N$ of $B\left(L_{2}(G)\right), C B_{N}^{\sigma, M}\left(B\left(L_{2}(G)\right)\right)$ denotes the space of all $N$-bimodule maps in $C B^{\sigma}\left(B\left(L_{2}(G)\right)\right)$ which map $M$ into $M$.

In this section, we study the range spaces of $L_{1}(G)$ and $A(G)$ under $\Theta_{r}$ and $\widehat{\Theta}$, respectively, i.e., we consider the corresponding representations of $L_{1}(G)$ and $A(G)$ in $C B^{\sigma}\left(B\left(L_{2}(G)\right)\right)$.

It is shown by Neufang-Ruan-Spronk [37, Theorem 3.6] that

$$
\Theta_{r}\left(L_{1}(G)\right)=C B_{V N(G)}^{\sigma,\left(L_{\infty}(G), C_{b}(G)\right)}\left(B\left(L_{2}(G)\right)\right),
$$

where $C_{b}(G)$ is the $C^{*}$-algebra of bounded continuous functions on $G$, and the superscript $\left(L_{\infty}(G), C_{b}(G)\right)$ is used to denote operators in $C B^{\sigma}\left(B\left(L_{2}(G)\right)\right)$ which map $L_{\infty}(G)$ into $C_{b}(G)$. By the definition of $\Theta_{r}$ (cf. [37]), we have

$$
\Theta_{r}\left(L_{1}(G)\right) \subseteq C B_{V N(G)}^{\sigma,\left(L_{\infty}(G), R U C(G)\right)}\left(B\left(L_{2}(G)\right)\right),
$$

where $R U C(G)$ is the $C^{*}$-algebra of bounded right uniformly continuous functions on $G$ (see below for the definition). Therefore, we also get

$$
\Theta_{r}\left(L_{1}(G)\right)=C B_{V N(G)}^{\sigma,\left(L_{\infty}(G), R U C(G)\right)}\left(B\left(L_{2}(G)\right)\right) .
$$

These results on $L_{1}(G)$ were obtained by some measure theoretic proofs.

We note that the space $C_{b}(G)$ may not be a natural object associated with the Banach algebra $L_{1}(G)$. Also, the above approaches to $L_{1}(G)$ seem hard to be adapted for $A(G)$. In the following, we study the two range space problems using a unified Banach algebraic approach.

First, we show that the group algebra $L_{1}(G)$ of any locally compact group $G$ is a Banach algebra of type $(M)$.

For $\mu \in M(G)$, let $R_{\mu}(h)=h * \mu\left(h \in L_{1}(G)\right)$. Then $R_{\mu} \in R M\left(L_{1}(G)\right)$. By a result of Wendel, each right multiplier of $L_{1}(G)$ is of this form. In this way, $L_{\infty}(G)$ is a left $M(G)$-module and $L_{\infty}(G)^{*}$ is a right $M(G)$-module with $\mu \cdot f=R_{\mu}^{*}(f)$ and 
$n \cdot \mu=R_{\mu}^{* *}(n)\left(f \in L_{\infty}(G)\right.$ and $\left.n \in L_{\infty}(G)^{*}\right)$. Note that if $h \in L_{1}(G)$ and $f \in L_{\infty}(G)$, then $h \cdot f=f * \check{h}$, where $\breve{h}(x)=h\left(x^{-1}\right)$. This is true because for all $\varphi \in L_{1}(G)$, we have

$$
\langle\varphi, h \cdot f\rangle=\langle\varphi * h, f\rangle=\int_{G} \int_{G} \varphi(t) f(s) \check{h}\left(s^{-1} t\right) d s d t=\langle\varphi, f * \check{h}\rangle .
$$

Let $\operatorname{LUC}(G)$ (resp. $R U C(G)$ ) be the $C^{*}$-algebra of bounded left (resp. right) uniformly continuous functions on $G$. That is,

$$
L U C(G)=\left\{f \in C_{b}(G) \text { : the map } a \mapsto{ }_{a} f \text { is continuous from } G \text { to } C_{b}(G)\right\},
$$

and

$$
R U C(G)=\left\{f \in C_{b}(G) \text { : the map } a \mapsto f_{a} \text { is continuous from } G \text { to } C_{b}(G)\right\},
$$

where ${ }_{a} f$ and $f_{a}$ denote the left and the right translates of $f$ by $a \in G$, respectively.

We note that $\operatorname{LUC}(G)=\mathfrak{C}_{r u}(G)$, the space of functions on $G$ which are uniformly continuous with respect to the right uniform structure on $G$ (see Hewitt-Ross [15]). By $[14,(32.45(\mathrm{~b}))], L_{1}(G) * L_{\infty}(G)=L U C(G)$. Therefore,

$$
L_{1}(G) \cdot L_{\infty}(G)=L_{\infty}(G) * L_{1}(G)^{\vee}=\left(L_{1}(G) * L_{\infty}(G)\right)^{\vee}=L U C(G)^{\vee}=R U C(G) .
$$

Similarly, one can define $L_{\mu} \in L M\left(L_{1}(G)\right)$, and each left multiplier of $L_{1}(G)$ is of the form $L_{\mu}$. Thus, both $L_{\infty}(G)$ and $L_{\infty}(G)^{*}$ are $M(G)$-bimodules. Comparing with $h \cdot f=f * \check{h}$, we have $f \cdot h=h^{*} * f$ for all $f \in L_{\infty}(G)$ and $h \in L_{1}(G)$, where $h^{*}(x)=\triangle\left(x^{-1}\right) h\left(x^{-1}\right)$, and $\triangle$ denotes the modular function of $G$. Therefore,

$$
L_{\infty}(G) \cdot L_{1}(G)=L_{1}(G) * L_{\infty}(G)=L U C(G) .
$$

Recall that $L_{1}(G)$ always has a BAI, and it has a sequential BAI precisely when $G$ is metrizable.

Proposition 8. Let $G$ be a locally compact group. Then $L_{1}(G)$ is a Banach algebra of type $(M)$. Therefore, for all $\mu \in M(G)$, the following assertions are equivalent:

(i) $\mu \in L_{1}(G)$.

(ii) There exists an $m \in L_{\infty}(G)^{*}$ such that $\mu \cdot n=m \cdot n$ for all $n \in L_{\infty}(G)^{*}$.

(iii) $L_{\infty}(G) \cdot \mu \subseteq L U C(G)$.

(iv) There exists an $m \in L_{\infty}(G)^{*}$ such that $n \cdot \mu=n \triangle m$ for all $n \in L_{\infty}(G)^{*}$.

(v) $\mu \cdot L_{\infty}(G) \subseteq R U C(G)$.

Proof. By Theorem 3(c), we may assume that $G$ is non-metrizable.

Let $\mu \in M(G)$. In the following, we identify $\mu$ with $L_{\mu} \in L M\left(L_{1}(G)\right)$, and we show the equivalence of (i), (ii), and (iii). Similar arguments will establish the equivalence of (i), (iv), and (v) with $\mu$ identified with $R_{\mu}$. 
Since $\mu$ is a regular finite Borel measure, there exists a $\sigma$-compact open subgroup $H$ of $G$ such that $\operatorname{supp} \mu \subseteq H$. Let $B=L_{1}(H)$. Since $A^{*} A=L U C(G)$ and $B^{*} B=L U C(H)$, condition (1) in Theorem 5 is satisfied. Obviously, condition (2) in Theorem 5 holds.

Let $\mathcal{N}$ be the family of compact normal subgroups $N$ of $H$ such that $H / N$ is metrizable. For each $N \in \mathcal{N}$, let $B_{N}=L_{1}(H / N)$. Then $\left\{B_{N}\right\}$ is a family of closed ideals in $B=L_{1}(H)$. To finish the proof, we show that conditions (I), (II), and (III) in Theorem 4 are satisfied for $B$.

(I) For each $N \in \mathcal{N}, B_{N}=L_{1}(H / N)$ is clearly WSC and has a sequential BAI, since $H / N$ is metrizable.

(II) For $N \in \mathcal{N}$ and $\varphi \in L_{1}(H)$, let $p_{N}(\varphi)=\lambda_{N} * \varphi$, where $\lambda_{N}$ is the normalized Haar measure on $N$. It is easy to see that $p_{N}$ is a $B_{N}$-bimodule projection from $L_{1}(H)$ onto $B_{N}=L_{1}(H / N)$.

(III) Let $\nu \in M(H)$. Again, we identify $\nu$ with $L_{\nu} \in L M\left(L_{1}(H)\right)$. Note that for each $N \in \mathcal{N},\left.\nu\right|_{B_{N}}=\nu * \lambda_{N}$. So, we assume that $\nu * \lambda_{N} \in L_{1}(H)$ for all $N \in \mathcal{N}$ and we prove below that there exists an $m \in L_{1}(H)$ such that $L_{\nu}=l_{m}$.

Clearly, $\mathcal{N}$ is a directed set ordered by reversed inclusion (cf. Hu [17, Theorem 2.2]), and $\left(\nu * \lambda_{N}\right)_{N \in \mathcal{N}}$ is bounded in $L_{1}(H)$. Let $m \in L_{1}(H)^{* *}$ be a weak*-cluster point of the net $\left(\nu * \lambda_{N}\right)_{N \in \mathcal{N}}$. Since $H$ is $\sigma$-compact, to obtain $m \in L_{1}(H)$, by Hu-Neufang [19, Corollary 3.3], one has only to prove that $m$ is weak ${ }^{*}$-sequentially continuous on $L_{\infty}(H)$.

For this purpose, let $\left(f_{n}\right)$ be a sequence in $L_{\infty}(H)$ such that $f_{n} \longrightarrow 0$ in the $\sigma\left(L_{\infty}(H), L_{1}(H)\right)$-topology. According to [17, Theorem 2.4 and Theorem 2.2], there exists an $N_{0} \in \mathcal{N}$ such that $f_{n} \in L_{\infty}\left(H / N_{0}\right)$ for all $n$. It follows that for all $n$ and $h \in L_{1}(H),\left\langle h * \lambda_{N_{0}}, f_{n}\right\rangle=\left\langle h, f_{n}\right\rangle$. Note that if $N \subseteq N_{0}$, then $\lambda_{N} * \lambda_{N_{0}}=\lambda_{N_{0}}$. Therefore, for all $n$ and $N \subseteq N_{0}$, we have

$$
\left\langle\nu * \lambda_{N}, f_{n}\right\rangle=\left\langle\nu * \lambda_{N} * \lambda_{N_{0}}, f_{n}\right\rangle=\left\langle\nu * \lambda_{N_{0}}, f_{n}\right\rangle
$$

since $\nu * \lambda_{N} \in L_{1}(H)$. So, $\left\langle m, f_{n}\right\rangle=\left\langle\nu * \lambda_{N_{0}}, f_{n}\right\rangle$ for all $n$. Again, by the fact that $\nu * \lambda_{N_{0}} \in L_{1}(H)$, we have

$$
\lim _{n}\left\langle m, f_{n}\right\rangle=\lim _{n}\left\langle\nu * \lambda_{N_{0}}, f_{n}\right\rangle=0
$$

Hence, $m$ is weak*-sequentially continuous on $L_{\infty}(H)$. The proof is complete.

Remark 9. Neufang showed in [32, Satz 3.7.7] the equivalence of (i) and (ii). A measure theoretic proof for the equivalence of (i) and (iii) ${ }^{\prime}$ is given by Hewitt-Ross [14, (35.13)] for compact $G$, and by Neufang-Ruan-Spronk [37, Lemma 3.5] for general $G$, where (iii)' is the condition: $\mu \cdot L_{\infty}(G) \subseteq C_{b}(G)$. 
We show below that Proposition 8 in fact holds for a general weighted convolution (Beurling) algebra $L_{1}(G, \omega)$ on a locally compact group $G$. Let us first recall some basic information on Beurling algebras. See Dales [3] and Dales-Lau [4] for details.

Let $\omega: G \longrightarrow(0, \infty)$ be a weight on $G$. That is, $\omega: G \longrightarrow(0, \infty)$ is continuous satisfying $\omega\left(e_{G}\right)=1$ and $\omega(s t) \leq \omega(s) \omega(t)(s, t \in G)$. Let

$$
L_{1}(G, \omega)=\left\{\varphi: \varphi \omega \in L_{1}(G)\right\} \text { and } L_{\infty}\left(G, \omega^{-1}\right)=\left\{f: f \omega^{-1} \in L_{\infty}(G)\right\} .
$$

Then $L_{1}(G, \omega)$ and $L_{\infty}\left(G, \omega^{-1}\right)$ are Banach spaces with norms defined by

$$
\|\varphi\|_{1, \omega}=\|\varphi \omega\|_{1} \text { and }\|f\|_{\infty, \omega}=\left\|f \omega^{-1}\right\|_{\infty}
$$

respectively. We have $L_{1}(G, \omega)^{*} \cong L_{\infty}\left(G, \omega^{-1}\right)$ via the duality

$$
\langle\varphi, f\rangle=\left\langle\varphi \omega, f \omega^{-1}\right\rangle_{L_{1}(G), L_{\infty}(G)}\left(\varphi \in L_{1}(G, \omega), f \in L_{\infty}\left(G, \omega^{-1}\right)\right) .
$$

Clearly, $L_{1}(G, \omega) \cong L_{1}(G)$ as Banach spaces. If we define the product $\cdot \omega$ on $L_{\infty}\left(G, \omega^{-1}\right)$ by $f \cdot \omega g=(f g) \omega^{-1}$, then $L_{\infty}\left(G, \omega^{-1}\right) \cong L_{\infty}(G)$ as von Neumann algebras. Let

$$
C_{0}\left(G, \omega^{-1}\right)=\left\{f \in L_{\infty}\left(G, \omega^{-1}\right): f \omega^{-1} \in C_{0}(G)\right\} .
$$

One can define the spaces $\operatorname{LUC}\left(G, \omega^{-1}\right)$ and $\operatorname{RUC}\left(G, \omega^{-1}\right)$ in a similar way. Then $C_{0}\left(G, \omega^{-1}\right), L U C\left(G, \omega^{-1}\right)$, and $R U C\left(G, \omega^{-1}\right)$ are $C^{*}$-subalgebras of $L_{\infty}\left(G, \omega^{-1}\right)$, *isomorphic to $C_{0}(G), L U C(G)$, and $R U C(G)$, respectively.

We know that if $G$ is amenable, then there exists a weight $\tilde{\omega}$ on $G$ such that $\tilde{\omega}(s) \geq 1$ $(s \in G)$ and $L_{1}(G, \tilde{\omega}) \cong L_{1}(G, \omega)$ as Banach algebras (cf. Dales-Lau [4, Theorem 7.44], and see below for the definition of the multiplication on $\left.L_{1}(G, \omega)\right)$. In the sequel, we assume that $\omega(s) \geq 1$ for all $s \in G$. Then $\omega^{-1} \in L_{\infty}(G)$ and $L_{1}(G, \omega) \subseteq L_{1}(G)$. Let $M(G, \omega)$ be the Banach space of complex-valued regular Borel measures $\mu$ on $G$ such that $\|\mu\|_{\omega}=\int_{G} \omega(s) d|\mu|(s)<\infty$. Then $M(G, \omega)$ is a linear subspace of $M(G)$, and $M(G, \omega) \cong C_{0}\left(G, \omega^{-1}\right)^{*}$ via the duality

$$
\langle f, \mu\rangle=\int_{G} f(s) d \mu(s)\left(f \in C_{0}\left(G, \omega^{-1}\right), \mu \in M(G, \omega)\right) .
$$

Furthermore, $M(G, \omega)$ is a Banach algebra with the convolution $*_{\omega}$ defined by

$$
\left\langle f, \mu *_{\omega} \nu\right\rangle=\int_{G} \int_{G} f(s t) d \mu(s) d \nu(t)\left(f \in C_{0}\left(G, \omega^{-1}\right), \mu, \nu \in M(G, \omega)\right) .
$$

It is known that $L_{1}(G, \omega)$ is a closed ideal in $M(G, \omega)$. Therefore, $L_{1}(G, \omega)$ is a Banach algebra, called the Beurling algebra on $G$ with weight $\omega$. We note that though $L_{1}(G, \omega) \cong L_{1}(G)$ as Banach spaces, they are not identified in general as Banach algebras.

Obviously, if $\omega=1$, then $L_{1}(G, \omega)=L_{1}(G), L_{\infty}\left(G, \omega^{-1}\right)=L_{\infty}(G)$, and $M(G, \omega)=$ $M(G)$. It is also known that for $A=L_{1}(G, \omega),\left\langle A^{*} A\right\rangle=L U C\left(G, \omega^{-1}\right),\left\langle A A^{*}\right\rangle=$ 
$R U C\left(G, \omega^{-1}\right)$, and the classical Wendel's theorem holds for $L_{1}(G, \omega)$. Naturally, $L_{\infty}\left(G, \omega^{-1}\right)$ and $L_{\infty}\left(G, \omega^{-1}\right)^{*}$ are Banach $M(G, \omega)$-bimodules.

As the predual of the von Neumann algebra $L_{\infty}\left(G, \omega^{-1}\right), L_{1}(G, \omega)$ is WSC. It is clear that the standard BAI in $L_{1}(G)$ with compact support is a BAI for $L_{1}(G, \omega)$. Therefore, if $G$ is metrizable, then $L_{1}(G, \omega)$ has a sequential BAI, and thus $L_{1}(G, \omega)$ is of type $(M)$. A close inspection and a slight modification of the proof of Proposition 8 shows that indeed every Beurling algebra $L_{1}(G, \omega)$ is of type $(M)$.

To be more precise, let $\mu \in M(G, \omega)(\subseteq M(G))$, and $H$ a $\sigma$-compact open subgroup of $G$ such that $\operatorname{supp} \mu \subseteq H$. We give below the main points that we should note and modify in the proof of Proposition 8 for the case of $L_{1}(G, \omega)$.

Firstly, we take a compact normal subgroup $N_{1}$ of $H$ such that $H / N_{1}$ is metrizable and $\omega^{-1} \in L_{\infty}\left(H / N_{1}\right)$ (cf. Hu [17, Theorem 2.4]). Secondly, the family $\mathcal{N}$ used in the proof of Proposition 8 should be replaced by its subfamily

$$
\mathcal{N}_{1}=\left\{N \in \mathcal{N}: N \subseteq N_{1}\right\}
$$

which is still a directed set with reversed inclusion (cf. [17, Theorem 2.2]). In this way, for all $\nu \in M(H, \omega)$, the net $\left(\nu *_{\omega} \lambda_{N}\right)_{N \in \mathcal{N}_{1}}$ is bounded in $M(H, \omega)$ :

$$
\left\|\nu *_{\omega} \lambda_{N}\right\|_{\omega} \leq\|\nu\|_{\omega} \sup _{s \in N_{1}} \omega(s) \text { for all } N \in \mathcal{N}_{1} .
$$

Thirdly, since $L_{1}(H, \omega) \cong L_{1}(H)$ as Banach spaces, Hu-Neufang [19, Corollary 3.3] (on the Mazur property of $\left.L_{1}(H)\right)$ holds for the Beurling algebra $L_{1}(H, \omega)$. Finally, one needs to apply [17, Theorem 2.4 and Theorem 2.2] to the sequence $\left(f_{n} \omega^{-1}\right)$ in $L_{\infty}(H)$, where $\left(f_{n}\right)_{n \geq 0}$ is a sequence in $L_{\infty}\left(H, \omega^{-1}\right)$ picked up for testing the weak ${ }^{*}$-sequential continuity of a weak* ${ }^{*}$ cluster point of the net $\left(\nu *_{\omega} \lambda_{N}\right)_{N \in \mathcal{N}_{1}}$ in $L_{1}(H, \omega)^{* *}$.

We are ready to state the Beurling algebra version of Proposition 8.

Theorem 10. Let $G$ be a locally compact group and $\omega$ a weight on $G$ with $\omega \geq 1$. Then $L_{1}(G, \omega)$ is a Banach algebra of type $(M)$. Therefore, for all $\mu \in M(G, \omega)$, the following assertions are equivalent:

(i) $\mu \in L_{1}(G, \omega)$.

(ii) There exists an $m \in L_{\infty}\left(G, \omega^{-1}\right)^{*}$ such that $\mu \cdot n=m \cdot n$ for all $n \in L_{\infty}\left(G, \omega^{-1}\right)^{*}$.

(iii) $L_{\infty}\left(G, \omega^{-1}\right) \cdot \mu \subseteq L U C\left(G, \omega^{-1}\right)$.

(iv) There exists an $m \in L_{\infty}\left(G, \omega^{-1}\right)^{*}$ such that $n \cdot \mu=n \triangle m$ for all $n \in L_{\infty}\left(G, \omega^{-1}\right)^{*}$.

(v) $\mu \cdot L_{\infty}\left(G, \omega^{-1}\right) \subseteq R U C\left(G, \omega^{-1}\right)$.

We turn now our attention to the Fourier algebra $A(G)$ of a locally compact group $G$. Since $A(G)$ is commutative, $\operatorname{LM}(A(G))=R M(A(G))$ as Banach spaces. Let $M A(G)$ 
denote the algebra of functions $\varphi$ on $G$ such that $\varphi f \in A(G)$ for all $f \in A(G)$. It is well-known that if $\varphi \in M A(G)$, then $\varphi \in C_{b}(G)$ and $\|\varphi\|_{\infty} \leq\|\varphi\|_{M A(G)}$, where

$$
\|\varphi\|_{M A(G)}=\sup \left\{\|\varphi f\|_{A(G)}: f \in A(G) \text { and }\|f\|_{A(G)} \leq 1\right\}<\infty .
$$

In this case, $m_{\varphi} \in L M(A(G))$ and $\left\|m_{\varphi}\right\|=\|\varphi\|_{M A(G)}$, where $m_{\varphi}: A(G) \longrightarrow A(G)$ is the map $f \mapsto \varphi f$. As observed by Losert [29], every (left) multiplier of $A(G)$ is of the form $m_{\varphi}$ for some $\varphi \in M A(G)$. Therefore, $L M(A(G)) \cong M A(G) \cong R M(A(G))$ as Banach algebras. Since $A(G)$ is a Banach $M A(G)$-bimodule, $V N(G)$ is naturally a Banach $M A(G)$-bimodule with the module actions given by

$$
\langle\varphi \cdot T, f\rangle=\langle T \cdot \varphi, f\rangle=\langle T, \varphi f\rangle
$$

$(\varphi \in M A(G), T \in V N(G), f \in A(G))$, and $V N(G)^{*}$ also becomes a Banach $M A(G)$ bimodule.

$\varphi \in M A(G)$ is called a completely bounded multiplier of $A(G)$ if $\left\|m_{\varphi}\right\|_{c b}<\infty$, where $A(G)$ has its natural operator space structure (cf. Ruan [41]). Let $M_{c b} A(G)$ denote all completely bounded multipliers on $A(G)$. Then $M_{c b} A(G)$ is a completely contractive Banach algebra and

$$
B(G) \subseteq M_{c b} A(G) \subseteq M A(G) \subseteq C_{b}(G)
$$

with all the three inclusion maps contractive. It is known that if $G$ is amenable, then $B(G)=M_{c b} A(G)=M A(G)$ isometrically (cf. De Cannière-Haagerup [6]).

$U C B(\widehat{G})$ will denote the closed linear span of $A(G) \cdot V N(G)$ in $V N(G)$. It is known that $U C B(\widehat{G})$ is the $C^{*}$-subalgebra of $V N(G)$ generated by elements of $V N(G)$ with compact support (cf. Granirer [13] and Lau [25]). $U C B(\widehat{G})=A(G) \cdot V N(G)$ if and only if $G$ is amenable (cf. Lau-Losert [26]), which is in turn equivalent to $A(G)$ having a BAI (cf. Leptin [28]). It can be seen that $A(G)$ has a sequential BAI if and only if $G$ is amenable and $\sigma$-compact (cf. Lau [25, Lemma 7.2]; see also Remark 15).

We obtain below the dual version of Proposition 8. The proof shows that when $G$ is amenable, $A(G)$ behaves even nicer than $L_{1}(G)$ in the sense that it satisfies the stronger conditions as stated in Theorem 4 instead of those in Theorem 5 .

Theorem 11. Let $G$ be an amenable locally compact group. Then $A(G)$ is a Banach algebra of type $(M)$. Therefore, for all $\varphi \in B(G)$, the following assertions are equivalent:

(i) $\varphi \in A(G)$.

(ii) There exists an $m \in A(G)^{* *}$ such that $\varphi \cdot n=m \cdot n$ for all $n \in A(G)^{* *}$.

(iii) $\varphi \cdot V N(G) \subseteq U C B(\widehat{G})$.

Proof. Since $G$ is amenable, $A(G)$ has a BAI. We prove that there exists a family of closed ideals in $A(G)$ satisfying (I), (II), and (III) in Theorem 4. 
Let $\mathcal{H}_{0}$ be the family of all $\sigma$-compact open subgroups of $G$. For each $H \in \mathcal{H}_{0}$, if we identify $A(H)$ with $\{f \in A(G): f=0$ on $G \backslash H\}$, then $A(H)$ can be treated as a closed ideal in $A(G)$. Let $p_{H}: A(G) \longrightarrow A(G)$ be the map $f \mapsto f \cdot 1_{H}\left(H \in \mathcal{H}_{0}\right)$, where $1_{H}$ is the characteristic function of $H$. Clearly, the family $\left\{\left(A(H), p_{H}\right)\right\}_{H \in \mathcal{H}_{0}}$ satisfies the conditions (I) and (II). By $\mathrm{Hu}[18$, Lemma 3.6], for any function $u$ on $G, u \in A(G)$ if $u \cdot 1_{H} \in A(H)$ for all $H \in \mathcal{H}_{0}$. Therefore, condition (III) is also satisfied.

Recall that, for $\mu \in M(G)$ and $f \in L_{\infty}(G), \Theta_{r}(\mu)(f)=\mu \cdot f$; for $\varphi \in M_{c b} A(G)$ and $T \in V N(G), \widehat{\Theta}(\varphi)(T)=\varphi \cdot T$ (cf. Neufang-Ruan-Spronk [37]). Applying Proposition 8 and Theorem 11, we have the following characterizations of the range spaces of the two algebras $L_{1}(G)$ and $A(G)$ under the representations $\Theta_{r}$ and $\widehat{\Theta}$, respectively. We point out that $A(G)$ can be identified with a norm closed ideal in $M_{c b} A(G)$ precisely when $G$ is amenable (cf. Losert [30] and Ruan [43]). We also note that (i) and (ii) below are dual to each other in the framework of locally compact quantum groups (cf. Theorem 14).

Theorem 12. Let $G$ be a locally compact group. Then

(i) $\Theta_{r}\left(L_{1}(G)\right)=C B_{V N(G)}^{\sigma,\left(L_{\infty}(G), R U C(G)\right)}\left(B\left(L_{2}(G)\right)\right)$;

(ii) $\widehat{\Theta}(A(G))=C B_{L_{\infty}(G)}^{\sigma,(V N(G), U C B(\widehat{G}))}\left(B\left(L_{2}(G)\right)\right)$ if $G$ is amenable.

Let $A_{c b}(G)$ denote the norm closure of $A(G)$ in $M_{c b} A(G)$ (cf. Forrest-Runde-Spronk $[9])$. Then $A_{c b}(G)$ is a closed ideal in $M_{c b} A(G)$ and $V N(G)$ is an $A_{c b}(G)$-bimodule. Since

$$
\|\varphi \cdot T\|_{V N(G)} \leq\|\varphi\|_{c b}\|T\|_{V N(G)} \text { for all } \varphi \in M_{c b} A(G) \text { and } T \in V N(G)
$$

we have $A_{c b}(G) \cdot V N(G) \subseteq U C B(\widehat{G})$, or equivalently, $\left\langle A_{c b}(G) \cdot V N(G)\right\rangle=U C B(\widehat{G})$. Therefore,

$$
\widehat{\Theta}\left(A_{c b}(G)\right) \subseteq C B_{L_{\infty}(G)}^{\sigma,(V N(G), U C B(\widehat{G}))}\left(B\left(L_{2}(G)\right)\right)
$$

Let

$$
A_{\widehat{\Theta}}(G)=\widehat{\Theta}^{-1}\left[C B_{L_{\infty}(G)}^{\sigma,(V N(G), U C B(\widehat{G}))}\left(B\left(L_{2}(G)\right)\right)\right]
$$

Then $A_{\widehat{\Theta}}(G)=\left\{\varphi \in M_{c b} A(G): \varphi \cdot V N(G) \subseteq U C B(\widehat{G})\right\}$, and $A_{\widehat{\Theta}}(G)$ is also a closed ideal in $M_{c b} A(G)$ with

$$
A(G) \subseteq A_{c b}(G) \subseteq A_{\widehat{\Theta}}(G) \subseteq M_{c b} A(G)
$$

Furthermore, $A_{c b}(G)$ is the smallest closed subalgebra $A$ of $M_{c b} A(G)$ containing $A(G)$ such that $\widehat{\Theta}(A) \subseteq C B_{L_{\infty}(G)}^{\sigma,(V N(G), U C B(\widehat{G}))}\left(B\left(L_{2}(G)\right)\right), A_{\widehat{\Theta}}(G)$ is the largest one among such closed subalgebras $A$ of $M_{c b} A(G)$, and we have

$$
\widehat{\Theta}\left(A_{\widehat{\Theta}}(G)\right)=C B_{L_{\infty}(G)}^{\sigma,(V N(G), U C B(\widehat{G}))}\left(B\left(L_{2}(G)\right)\right) .
$$


Clearly, $A_{\widehat{\Theta}}(G)=M_{c b} A(G)$ if and only if $G$ is compact. Theorem 12(ii) shows that if $G$ is amenable, then $A_{\widehat{\Theta}}(G)=A(G)$, which obviously implies that $A_{c b}(G)=A(G)$ and $A_{\widehat{\Theta}}(G)=A_{c b}(G)$. As noted in the paragraph preceding Theorem 12, if $A_{c b}(G)=A(G)$, then $G$ is amenable. Therefore, we have the following

Corollary 13. Let $G$ be a locally compact group. Then the following statements are equivalent:

(i) $G$ is amenable.

(ii) $A_{\widehat{\Theta}}(G)=A(G)$.

(iii) $A_{c b}(G)=A(G)$.

It may be natural to conjecture that $A_{\widehat{\Theta}}(G)=A_{c b}(G)$ would also force $G$ to be amenable.

As mentioned earlier (cf. [37, Theorem 3.6]), we have

$$
\Theta_{r}\left(L_{1}(G)\right)=C B_{V N(G)}^{\sigma,\left(L_{\infty}(G), C_{b}(G)\right)}\left(B\left(L_{2}(G)\right)\right) .
$$

Related to this result, the right side version of Dzinotyiweyi-van Rooij [5, Theorem] implies that

$$
\Theta_{r}\left(L_{1}(G)\right)=C B_{V N(G)}^{\sigma,\left(C_{b}(G), R U C(G)\right)}\left(B\left(L_{2}(G)\right)\right) \text { if } G \text { is non-compact. }
$$

It may be interesting to consider possible dual versions of these results. Note that $C_{b}(G)$ is the multiplier $C^{*}$-algebra of $C_{0}(G)$. It may be a natural conjecture that for the case of $A(G)$, the above $C_{b}(G)$ should be replaced by $M C_{\lambda}^{*}(G)$, the multiplier $C^{*}$-algebra of the reduced group $C^{*}$-algebra $C_{\lambda}^{*}(G)$ of $G$ (cf. [37, Remark 4.9]).

At the end of this section, we describe briefly how Theorem 12 can be extended to locally compact quantum groups.

Let $\mathbb{G}$ be a co-amenable locally compact quantum group. Generalizing and unifying the representation theorems in [32] and [37] on locally compact groups, Junge, Neufang and Ruan showed in [21, Theorem 6.3] that there exists a completely isometric algebra isomorphism $\pi$ from $M_{c b}^{r}\left(L_{1}(\mathbb{G})\right)$ onto $C B_{L_{\infty}(\mathbb{\mathbb { G }})}^{\sigma, L_{\infty}(\mathbb{G})}\left(B\left(L_{2}(\mathbb{G})\right)\right)$. Here, $M_{c b}^{r}\left(L_{1}(\mathbb{G})\right)$ is the algebra of completely bounded right multipliers of $L_{1}(\mathbb{G})$, which is introduced by JungeNeufang-Ruan [21] and is defined as the set of all $q \in L_{\infty}(\widehat{\mathbb{G}})^{\prime}$ such that $\rho(f) q \in \rho\left(L_{1}(\mathbb{G})\right)$ for all $f \in L_{1}(\mathbb{G})$ and the induced map

$$
m_{q}^{r}: L_{1}(\mathbb{G}) \longrightarrow L_{1}(\mathbb{G}), f \longmapsto \rho^{-1}(\rho(f) q) \text { is completely bounded, }
$$

where $L_{\infty}(\widehat{\mathbb{G}})^{\prime}$ is the commutant of $L_{\infty}(\widehat{\mathbb{G}})$ in $B\left(L_{2}(\mathbb{G})\right)$, and $\rho: L_{1}(\mathbb{G}) \longrightarrow L_{\infty}(\widehat{\mathbb{G}})^{\prime}$ is the right regular representation of $\mathbb{G}$. 
It is shown in [21] that for each $q \in M_{c b}^{r}\left(L_{1}(\mathbb{G})\right)$, the map $m_{q}^{r}: f \mapsto \rho^{-1}(\rho(f) q)$ on $L_{1}(\mathbb{G})$ does define an element of $R M\left(L_{1}(\mathbb{G})\right)$ satisfying

$$
\pi(q)(x)=\left(m_{q}^{r}\right)^{*}(x) \text { for all } x \in L_{\infty}(\mathbb{G}) .
$$

Naturally, $L_{1}(\mathbb{G})$ is identified with a right (actually two sided) ideal in $M_{c b}^{r}\left(L_{1}(\mathbb{G})\right.$ ) via $\rho$, and for all $f \in L_{1}(\mathbb{G}), m_{\rho(f)}^{r}$ is just the right multiplier on $L_{1}(\mathbb{G})$ induced by $f$. Therefore, we have

$$
\pi(\rho(f))(x)=f \cdot x \text { for all } f \in L_{1}(\mathbb{G}) \text { and } x \in L_{\infty}(\mathbb{G}) .
$$

See Junge-Neufang-Ruan [21] for more information on the algebra $M_{c b}^{r}\left(L_{1}(\mathbb{G})\right)$ and the representation $\pi$.

Following the classical case, let us define

$$
R U C(\mathbb{G})=\left\langle L_{1}(\mathbb{G}) \cdot L_{\infty}(\mathbb{G})\right\rangle
$$

Then $R U C(\mathbb{G})=L_{1}(\mathbb{G}) \cdot L_{\infty}(\mathbb{G})$, since $L_{1}(\mathbb{G})$ has a BAI. Obviously, if $f \in L_{1}(\mathbb{G})$, then $\pi(\rho(f))\left(L_{\infty}(\mathbb{G})\right)=f \cdot L_{\infty}(\mathbb{G}) \subseteq R U C(\mathbb{G})$. So, under the identification $L_{1}(\mathbb{G}) \cong$ $\rho\left(L_{1}(\mathbb{G})\right)$, we have

$$
\pi\left(L_{1}(\mathbb{G})\right) \subseteq C B_{L_{\infty}(\widehat{\mathbb{G}})}^{\sigma,\left(L_{\infty}(\mathbb{G}), R U C(\mathbb{G})\right)}\left(B\left(L_{2}(\mathbb{G})\right)\right)
$$

Conversely, assume that $q \in M_{c b}^{r}\left(L_{1}(\mathbb{G})\right)$ and $\pi(q)\left(L_{\infty}(\mathbb{G})\right) \subseteq R U C(\mathbb{G})$. Then

$$
\left(m_{q}^{r}\right)^{*}\left(L_{\infty}(\mathbb{G})\right) \subseteq R U C(\mathbb{G})
$$

If $L_{1}(\mathbb{G})$ is of type $(R M)$, then, by Definition 6 , there exists an $f_{0} \in L_{1}(\mathbb{G})$ such that

$$
m_{q}^{r}(f)=\rho^{-1}(\rho(f) q)=f \star f_{0} \text { for all } f \in L_{1}(\mathbb{G})
$$

In this case,

$$
\rho(f) q=\rho(f) \rho\left(f_{0}\right) \text { for all } f \in L_{1}(\mathbb{G})
$$

and, since $\rho\left(L_{1}(\mathbb{G})\right)$ is weak*-dense in $L_{\infty}(\widehat{\mathbb{G}})^{\prime}$, we have $q=\rho\left(f_{0}\right)$, i.e., $q \in L_{1}(\mathbb{G})$.

It is routine to check that an involutive Banach algebra $A$ is of type $(R M)$ if and only if $A$ is of type $(L M)$, and hence if and only if $A$ is of type $(M)$. Therefore, $L_{1}(\mathbb{G})$ is of type $(R M)$ if and only if it is of type $(M)$, since $L_{1}(\mathbb{G})$ is an involutive Banach algebra (cf. Section 2).

Summarizing the above discussions, we get a locally compact quantum group version of Theorem 12 as stated below. 
Theorem 14. Let $\mathbb{G}$ be a co-amenable locally compact quantum group such that $L_{1}(\mathbb{G})$ is of type $(M)$. Let $\pi$ be the completely isometric algebra isomorphism from $M_{c b}^{r}\left(L_{1}(\mathbb{G})\right)$ onto $C B_{L_{\infty}(\widetilde{\mathbb{G}})}^{\sigma, L_{\infty}(\mathbb{G})}\left(B\left(L_{2}(\mathbb{G})\right)\right)$ as above. Then

$$
\pi\left(L_{1}(\mathbb{G})\right)=C B_{L_{\infty}(\mathbb{\mathbb { G }})}^{\sigma,\left(L_{\infty}(\mathbb{G}), R U C(\mathbb{G})\right)}\left(B\left(L_{2}(\mathbb{G})\right)\right) .
$$

In particular, this assertion holds for every co-amenable locally compact quantum group $\mathbb{G}$ with $L_{1}(\mathbb{G})$ separable.

Remark 15. Let $G$ be a locally compact group. It is known that $L_{1}(G)$ always has a BAI with cardinality dominated by $\chi(G)$ (the local weight of $G$ ). And when $G$ is amenable, $A(G)$ has a BAI of cardinality no more than $\kappa(G)$ (the compact covering number of $G)$. In fact, it can be shown that the above two cardinals $\chi(G)$ and $\kappa(G)$ are the greatest lower bounds for the cardinality of any BAI in $L_{1}(G)$ and $A(G)$ (with $G$ amenable), respectively, and they are attained. Therefore, if $\mathbb{G}=\mathbb{G}_{a}$ or $\mathbb{G}=\mathbb{G}_{s}$ is a co-amenable locally compact quantum group, then $L_{1}(\mathbb{G})$ has a BAI with cardinality less than or equal to $\operatorname{dec}\left(L_{\infty}(\widehat{\mathbb{G}})\right)$, the decomposability number of the von Neumann algebra $L_{\infty}(\widehat{\mathbb{G}})$ (cf. Hu-Neufang [19]).

It is interesting to know whether this assertion is true for all co-amenable locally compact quantum groups. If so, then the description on the image $\pi\left(L_{1}(\mathbb{G})\right)$ in Theorem 14 would be valid for all co-amenable co- $\sigma$-finite locally compact quantum groups $\mathbb{G}$, where $\mathbb{G}$ is called co- $\sigma$-finite if $L_{\infty}(\widehat{\mathbb{G}})$ is a $\sigma$-finite von Neumann algebra.

Of course, it is even more interesting to know whether for all co-amenable locally compact quantum groups $\mathbb{G}, L_{1}(\mathbb{G})$ is of type $(M)$, which is true when $\mathbb{G}=\mathbb{G}_{a}$ or $\mathbb{G}=\mathbb{G}_{s}$ (cf. Remark 7(a)). If it would be the case, then the assertion in Theorem 14 would be true for all co-amenable locally compact quantum groups.

\section{Multipliers, characterizations of $A$ inside $A^{* *}$, and topological}

\section{CENTRES}

For the class of Banach algebras $A$ considered in Section 3, with the help of Theorem 5 on multipliers, we first give in Theorem 18 below some criteria characterizing elements of $A$ under the canonical embedding $A \hookrightarrow A^{* *}$. The formulation of Theorem 18 is motivated by Lau-Ülger [27, Theorem 5.4], where they considered the case when $A=L_{1}(G)$ (see Remark 20 below). In [27], Lau-Ülger further asked whether their Theorem 5.4 extends to $A(G)$ of an amenable locally compact group $G$ (cf. [27, question $6 \mathrm{~h}$ )]).

In the sequel, we need the notation $\mathcal{E}$ for the set of all mixed identities of $A^{* *}$. That is,

$$
\mathcal{E}=\left\{E \in A^{* *}: n \cdot E=E \triangle n=n \text { for all } n \in A^{* *}\right\}
$$


It is known that $E \in \mathcal{E}$ if and only if $E$ is a weak*-cluster point of a BAI of $A$ in $A^{* *}$ (cf. Dales [3, Proposition 2.9.16(iii)] and Palmer [38, Proposition 5.1.8(a)]). Also, it is easy to see that if $n_{1} \in Z_{t}^{(l)}\left(A^{* *}\right)$ and $n_{2} \in Z_{t}^{(r)}\left(A^{* *}\right)$, then $n_{1} \triangle E=n_{1}$ and $E \cdot n_{2}=n_{2}$ for all $E \in \mathcal{E}$.

We start with two results on general Banach algebras.

Lemma 16. Let $A$ be a Banach algebra and $m \in A^{* *}$. Consider the following statements.

(i) $m \cdot A \subseteq A$ and $A^{*} \triangle m \subseteq\left\langle A^{*} A\right\rangle$.

(ii) $\lambda^{m}=\mu^{* *}$ for some $\mu \in L M(A)$ satisfying $\mu^{*}\left(A^{*}\right) \subseteq\left\langle A^{*} A\right\rangle$.

(iii) $m=m_{0}+r$ with $m_{0} \in Z_{t}^{(l)}\left(A^{* *}\right)$ satisfying $m_{0} \cdot A \subseteq A$ and $r \in\left\langle A A^{*}\right\rangle^{\perp}$.

(i) $A \cdot m \subseteq A$ and $m \cdot A^{*} \subseteq\left\langle A A^{*}\right\rangle$.

(ii) $)^{\prime} \rho_{m}=\mu^{* *}$ for some $\mu \in R M(A)$ satisfying $\mu^{*}\left(A^{*}\right) \subseteq\left\langle A A^{*}\right\rangle$.

(iii)' $m=m_{0}+r$ with $m_{0} \in Z_{t}^{(r)}\left(A^{* *}\right)$ satisfying $A \cdot m_{0} \subseteq A$ and $r \in\left\langle A^{*} A\right\rangle^{\perp}$.

Then

(I) (iii) $\Longrightarrow($ i $) \Longleftrightarrow\left(\right.$ ii), and (iii) $\Longrightarrow(i)^{\prime} \Longleftrightarrow(\text { ii })^{\prime}$.

(II) If $A$ has a BAI, then (i) $\Longleftrightarrow($ ii $) \Longleftrightarrow($ iii $)$, and (i) $\Longleftrightarrow(\text { ii })^{\prime} \Longleftrightarrow(\text { iii })^{\prime}$.

Proof. (I) It is routine to check that $[(\mathrm{i}) \Longleftrightarrow(\mathrm{ii})]$ and $\left[(\mathrm{i})^{\prime} \Longleftrightarrow(\mathrm{ii})^{\prime}\right]$.

Assume (iii) holds. It is easy to see that $r \cdot A=\{0\}$ and $A^{*} \triangle r=\{0\}$. Thus, $m \cdot A=m_{0} \cdot A \subseteq A$ and, by Dales-Lau [4, Proposition 2.20], we have

$$
A^{*} \triangle m=A^{*} \triangle m_{0} \subseteq\left\langle A^{*} A\right\rangle .
$$

Therefore, (i) is true. The proof of $\left[(\text { iii })^{\prime} \Longrightarrow(\mathrm{i})^{\prime}\right]$ is similar.

(II) Assume $A$ has a BAI. We prove that (ii) $\Longrightarrow\left(\right.$ iii). [(ii) $\left.{ }^{\prime} \Longrightarrow(\text { iii })^{\prime}\right]$ can be proved in a similar way.

So, we suppose $\lambda^{m}=\mu^{* *}$ for some $\mu \in L M(A)$ satisfying $\mu^{*}\left(A^{*}\right) \subseteq\left\langle A^{*} A\right\rangle$. By Theorem 3(b), $\mu^{* *}=\lambda_{m_{0}}$ for some $m_{0} \in A^{* *}$. In particular, the map $n \mapsto m_{0} \cdot n=\mu^{* *}(n)$ is weak*-weak* continuous on $A^{* *}$. Hence, $m_{0} \in Z_{t}^{(l)}\left(A^{* *}\right)$. Since, for all $a \in A$, $m_{0} \cdot a=\mu(a)=m \cdot a$, we have $m_{0} \cdot A=m \cdot A \subseteq A$. Let $r=m-m_{0}$. Clearly, $r \in\left\langle A A^{*}\right\rangle^{\perp}$. Therefore, (iii) holds.

Theorem 17. Let $A$ be a Banach algebra and $m \in A^{* *}$. Consider the following statements.

(i) $m \cdot A \subseteq A$ and $m \in Z_{t}^{(l)}\left(A^{* *}\right)$.

(ii) $\lambda_{m}=\mu^{* *}$ for some $\mu \in L M(A)$.

(iii) $m \cdot A \subseteq A, A^{*} \triangle m \subseteq\left\langle A^{*} A\right\rangle$, and $m \triangle E=m$ for some $E \in \mathcal{E}$.

$(i)^{\prime} A \cdot m \subseteq A$ and $m \in Z_{t}^{(r)}\left(A^{* *}\right)$. 
$(\text { ii) })^{\prime} \rho^{m}=\mu^{* *}$ for some $\mu \in R M(A)$.

(iii) $A \cdot m \subseteq A, m \cdot A^{*} \subseteq\left\langle A A^{*}\right\rangle$, and $E \cdot m=m$ for some $E \in \mathcal{E}$.

Then

(I) (i) $\Longleftrightarrow$ (ii), and (i) $\Longleftrightarrow($ ii)'.

(II) If $A$ has a BAI, then (i) $\Longleftrightarrow\left(\right.$ ii) $\Longleftrightarrow\left(\right.$ iii), and (i) $\Longleftrightarrow(\text { ii })^{\prime} \Longleftrightarrow($ (iii) .

Proof. (I) Note that $\lambda_{m}=\lambda^{m}$ if and only if $m \in Z_{t}^{(l)}\left(A^{* *}\right)$. Also, $A^{*} \triangle Z_{t}^{(l)}\left(A^{* *}\right) \subseteq$ $\left\langle A^{*} A\right\rangle$ (cf. [4, Proposition 2.20]). Clearly, if $\lambda_{m}=\mu^{* *}$ for some $\mu \in L M(A)$, then $m \in Z_{t}^{(l)}\left(A^{* *}\right)$. Therefore, applying Lemma 16(I), we have (i) $\Longleftrightarrow$ (ii). Similarly, we have (i) $\Longleftrightarrow(\text { ii })^{\prime}$.

(II) Assume $A$ has a BAI. Obviously, (i) $\Longrightarrow$ (iii), and (i) $)^{\prime} \Longrightarrow\left(\right.$ (iii) ${ }^{\prime}$. We prove that (iii) $\Longrightarrow\left(\right.$ i). The proof of $\left[(\text { iii })^{\prime} \Longrightarrow(\text { i })^{\prime}\right]$ is similar.

Suppose (iii) holds. By Lemma 16(II), $m=m_{0}+r$ for some $m_{0} \in Z_{t}^{(l)}\left(A^{* *}\right)$ and $r \in\left\langle A A^{*}\right\rangle^{\perp}$. Since $r \triangle E=0$, we have

$$
m=m \triangle E=m_{0} \triangle E=m_{0} \cdot E=m_{0} \in Z_{t}^{(l)}\left(A^{* *}\right) .
$$

Therefore, (i) is true.

We note that the equivalence of (i) and (iii) in Theorem 17(II) can also be derived from Lau-Ülger [27, Theorem 5.1] (see Remark 20 below on the interchanging of the words "some" and "each").

By Theorem 5 and Theorem 17 and omitting the parts on multipliers, we have the following theorem, which nicely characterizes a normal element of $A^{* *}$ in terms of its behaviour on $A, A^{*}$, and $A^{* *}$.

Theorem 18. Let $A$ be a Banach algebra and $m \in A^{* *}$. Consider the following statements.

(i) $m \in A$.

(ii) $m \cdot A \subseteq A$ and $m \in Z_{t}^{(l)}\left(A^{* *}\right)$.

(iii) $m \cdot A \subseteq A, A^{*} \triangle m \subseteq\left\langle A^{*} A\right\rangle$, and $m \triangle E=m$ for some $E \in \mathcal{E}$.

(iv) $A \cdot m \subseteq A, m \cdot A^{*} \subseteq\left\langle A A^{*}\right\rangle$, and $E \cdot m=m$ for some $E \in \mathcal{E}$.

(v) $A \cdot m \subseteq A$ and $m \in Z_{t}^{(r)}\left(A^{* *}\right)$.

Then (i) $\Longleftrightarrow$ (ii) $\Longleftrightarrow$ (iii) if $A$ is of type $(L M)$, and (i) $\Longleftrightarrow$ (iv) $\Longleftrightarrow$ (v) if $A$ is of type $(R M)$.

In particular, (i) - (v) are equivalent if $A$ is of type $(M)$.

Combining Theorem 18 with Theorem 10 and Theorem 11, respectively, we get below a generalization of [27, Theorem 5.4] and its dual version by using multipliers through our unified Banach algebraic approach. 
Corollary 19. The assertions (i)-(v) in Theorem 18 are equivalent for all $m \in A^{* *}$, where

(1) $A=L_{1}(G, \omega)$ for any locally compact group $G$ with $\omega \geq 1$, or

(2) $A=A(G)$ for any amenable locally compact group $G$.

Remark 20. Lau-Ülger [27, Theorem 5.4] states that for $A=L_{1}(G)$ for any locally compact group $G$ and $m \in A^{* *}, m \in A$ if and only if $A \cdot m \subseteq A, m \cdot A^{*} \subseteq\left\langle A A^{*}\right\rangle$, and $E \cdot m=m$ for each $E \in \mathcal{E}$. We note here that when the condition " $m \cdot A^{*} \subseteq\left\langle A A^{*}\right\rangle$ " is satisfied, $E_{1} \cdot m=E_{2} \cdot m$ for all $E_{1}, E_{2} \in \mathcal{E}$. Therefore, the word "each" in [27, Theorem 5.4] can be replaced by "some" as shown in Theorem 18(iv).

It is noted by Dales-Lau in [4, Example 4.3] that [27, Theorem 5.4] "may not be true" due to the fact that $[27$, Lemma 5.3] is "not quite precise".

In fact, the proof of [27, Theorem 5.4] needs $L_{1}(G)$ to possess property (*) of Godefroy (cf. [12, p.155]). By Neufang [36, Theorem 2.18, Theorem 2.26, and Remark 2.19], this hypothesis on $L_{1}(G)$ is equivalent to the compact covering number $\kappa(G)$ of $G$ being a non-measurable cardinal. Therefore, the proof of [27, Theorem 5.4] is valid under the assumption that $\kappa(G)$ is non-measurable (see Neufang [33, p.166]).

Theorem 18 and Corollary 19 show that a more general and abstract form of [27, Theorem 5.4] is indeed true. See Corollary 28 and Corollary 30 below for even stronger forms of $[27$, Theorem 5.4].

Next, we show that for some Banach algebras, the middle condition in Theorem 18 (iii) and (iv) can be removed. In this case, however, we do need that the equalities " $m \triangle E=m$ " and " $E \cdot m=m$ " as stated in Theorem 18(iii) and (iv), respectively, hold for all $E \in \mathcal{E}$ (cf. Remark 20).

We begin with a lemma on multipliers, some forms of which may be known, but we could not find a reference for the form we need here.

Lemma 21. Let $A$ be a Banach algebra.

(1) Let $\mu \in L M(A)$. If $A$ has a bounded right approximate identity, then $\left[\mu^{*}\left(A^{*}\right) \subseteq\left\langle A^{*} A\right\rangle\right]$ if and only if $\left[\mu^{* *}\left(E_{1}\right)=\mu^{* *}\left(E_{2}\right)\right.$ for all $\left.E_{1}, E_{2} \in \mathcal{E}_{R}\right]$, where $\mathcal{E}_{R}$ is the set of right identities of $\left(A^{* *}, \cdot\right)$.

(2) Let $\mu \in R M(A)$. If $A$ has a bounded left approximate identity, then $\left[\mu^{*}\left(A^{*}\right) \subseteq\left\langle A^{*} A\right\rangle\right]$ if and only if $\left[\mu^{* *}\left(E_{1}\right)=\mu^{* *}\left(E_{2}\right)\right.$ for all $\left.E_{1}, E_{2} \in \mathcal{E}_{L}\right]$, where $\mathcal{E}_{L}$ is the set of left identities of $\left(A^{* *}, \triangle\right)$.

(3) If $A$ has a BAI and $\left\langle A^{*} A\right\rangle=\left\langle A A^{*}\right\rangle$, then (1) and (2) hold with both $\mathcal{E}_{R}$ and $\mathcal{E}_{L}$ replaced by $\mathcal{E}$. 
(4) If $A$ is WSC with a sequential BAI $\left(e_{n}\right)$, then (1) and (2) hold with both $\mathcal{E}_{R}$ and $\mathcal{E}_{L}$ replaced by the set of weak*-cluster points of $\left(e_{n}\right)$ in $A^{* *}$.

Proof. We first prove assertion (1). The proof of (2) is similar.

Assume that $\mu \in L M(A)$ and $A$ has a bounded right approximate identity.

Suppose $\mu^{*}\left(A^{*}\right) \subseteq\left\langle A^{*} A\right\rangle=A^{*} A$, and $E_{1}, E_{2} \in \mathcal{E}_{R}$. Note that $\left.E_{1}\right|_{A^{*} A}=\left.E_{2}\right|_{A^{*} A}$. Then, for all $f \in A^{*}$, we have

$$
\left\langle f, \mu^{* *}\left(E_{1}\right)\right\rangle=\left\langle\mu^{*}(f), E_{1}\right\rangle=\left\langle\mu^{*}(f), E_{2}\right\rangle=\left\langle f, \mu^{* *}\left(E_{2}\right)\right\rangle .
$$

Therefore, $\mu^{* *}\left(E_{1}\right)=\mu^{* *}\left(E_{2}\right)$.

Conversely, suppose $\mu^{* *}\left(E_{1}\right)=\mu^{* *}\left(E_{2}\right)$ for all $E_{1}, E_{2} \in \mathcal{E}_{R}$. Let $f \in A^{*}$ and $r \in$ $\left\langle A^{*} A\right\rangle^{\perp}$. Fix an $E \in \mathcal{E}_{R}$. Then $r+E \in \mathcal{E}_{R}$. By the assumption, $\mu^{* *}(r+E)=\mu^{* *}(E)$, and thus,

$$
\left\langle\mu^{*}(f), r\right\rangle=\left\langle f, \mu^{* *}(r+E)\right\rangle-\left\langle f, \mu^{* *}(E)\right\rangle=0 .
$$

It follows that $\mu^{*}(f) \in\left\langle A^{*} A\right\rangle$. Therefore, $A^{*} \triangle m \subseteq\left\langle A^{*} A\right\rangle$.

Clearly, the above proof shows that (3) holds.

To prove (4), we assume $A$ is WSC with a sequential BAI $\left(e_{n}\right)$ and $\mu \in L M(A)$ (the proof for the case $\mu \in R M(A)$ follows from a similar argument).

Let $\mathcal{E}_{0}$ be the set of all weak* ${ }^{*}$-cluster points of $\left(e_{n}\right)$ in $A^{* *}$. Suppose $\mu^{* *}\left(E_{1}\right)=\mu^{* *}\left(E_{2}\right)$ for all $E_{1}, E_{2} \in \mathcal{E}_{0}$. Then $\left(\mu\left(e_{n}\right)\right)$ is a weakly Cauchy sequence in $A$. Since $A$ is WSC, $\mu\left(e_{n}\right) \longrightarrow a_{0}$ weakly in $A$ for some $a_{0} \in A$. Therefore, $\mu^{*}(f)=f \cdot a_{0}$ for all $f \in A^{*}$, and hence $\mu^{*}\left(A^{*}\right) \subseteq\left\langle A^{*} A\right\rangle$.

Note that if $m \in A^{* *}$ and $m \cdot A \subseteq A$ (resp. $A \cdot m \subseteq A$ ), then $m$ defines a left (resp. right) multiplier on $A$. Therefore, combining Theorem 18 and Lemma 21, we have

Corollary 22. Let $A$ be a Banach algebra of type $(M)$ and $m \in A^{* *}$. Then the following statements are equivalent:

(i) $m \in A$.

(ii) $m \cdot A \subseteq A$ and $m \in Z_{t}^{(l)}\left(A^{* *}\right)$.

(iii) $m \cdot A \subseteq A$ and $m \triangle E=m$ for all $E \in \mathcal{E}_{R}$.

(iv) $A \cdot m \subseteq A$ and $E \cdot m=m$ for all $E \in \mathcal{E}_{L}$.

(v) $A \cdot m \subseteq A$ and $m \in Z_{t}^{(r)}\left(A^{* *}\right)$.

In addition, if either $\left\langle A^{*} A\right\rangle=\left\langle A A^{*}\right\rangle$ or $A$ has a sequential BAI, then the sets $\mathcal{E}_{R}$ and $\mathcal{E}_{L}$ can be replaced by $\mathcal{E}$.

It can be seen that if $A$ has a BAI and $A A^{* *} \subseteq A$ (resp. $A^{* *} A \subseteq A$ ), then $\left\langle A^{*} A\right\rangle \subseteq$ $\left\langle A A^{*}\right\rangle$ (resp. $\left\langle A A^{*}\right\rangle \subseteq\left\langle A^{*} A\right\rangle$ ). Hence, $\left\langle A^{*} A\right\rangle=\left\langle A A^{*}\right\rangle$ if $A$ has a BAI and $A$ is an ideal in $A^{* *}$. Obviously, we also have $\left\langle A^{*} A\right\rangle=\left\langle A A^{*}\right\rangle$ if $A$ has a central BAI. In particular, 
this is the case if $A$ is a commutative Banach algebra with a BAI. Therefore, by Lemma 21(3) and Theorem 11, we reach the following characterization of $A(G)$ inside $B(G)$ when $G$ is amenable.

Corollary 23. Let $G$ be an amenable locally compact group and $\varphi \in B(G)$. Then

$$
[\varphi \in A(G)] \text { if and only if }\left[E_{1} \cdot \varphi=E_{2} \cdot \varphi \text { for all } E_{1}, E_{2} \in \mathcal{E}\right]
$$

Clearly, by Corollary 22, we can deduce the following result of Miao, which answers positively the open question $6 \mathrm{~h}$ ) in [27] in a stronger form (cf. the first paragraph of this section).

Corollary 24. (Miao [31, Theorem 3.2]) Let $G$ be an amenable locally compact group and $m \in A(G)^{* *}$. Then

$$
[m \in A(G)] \text { if and only if }[A(G) \cdot m \subseteq A(G) \text { and } E \cdot m=m \text { for all } E \in \mathcal{E}] \text {. }
$$

To prove Corollary 23 and Corollary 24 for a more general Banach algebra, we need the following technical lemma on mixed identities. The case for right identities of $\left(A^{* *}, \cdot\right)$ is included in the proof of Miao [31, Theorem 2.3].

Lemma 25. Let $A$ be a Banach algebra and $I$ a closed ideal in $A$. Assume that there exists an A-bimodule projection $p$ from $A$ onto $I$. If $E \in \mathcal{E}, E_{0} \in \mathcal{E}_{I}$, and $E^{\prime}=E-$ $(\tau p)^{* *}(E)+\tau^{* *}\left(E_{0}\right)$, then $E^{\prime} \in \mathcal{E}$ and $p^{* *}\left(E^{\prime}\right)=E_{0}$, where $\mathcal{E}_{I}$ is the set of mixed identities of $I$, and $\tau: I \longrightarrow A$ is the inclusion map.

Proof. Let $E \in \mathcal{E}$ and $E_{0} \in \mathcal{E}_{I}$. By the definition of the module structures on $A^{*}$, it can be seen that, for all $a \in A$ and $f \in A^{*}$,

$$
\left\langle(\tau p)^{* *}(E), f \cdot a\right\rangle=\langle p(a), f\rangle=\left\langle\tau^{* *}\left(E_{0}\right), f \cdot a\right\rangle,
$$

and

$$
\left\langle a \cdot f,(\tau p)^{* *}(E)\right\rangle=\langle p(a), f\rangle=\left\langle a \cdot f, \tau^{* *}\left(E_{0}\right)\right\rangle .
$$

It follows that, for all $n \in A^{* *}$, we have

$$
n \cdot(\tau p)^{* *}(E)-n \cdot \tau^{* *}\left(E_{0}\right)=0,
$$

and

$$
(\tau p)^{* *}(E) \triangle n-\tau^{* *}\left(E_{0}\right) \triangle n=0 .
$$

Hence, $n \cdot E^{\prime}=n=E^{\prime} \triangle n$ for all $n \in A^{* *}$, i.e., $E^{\prime} \in \mathcal{E}$.

Since $p \tau=i d$, we have $p^{* *} \tau^{* *}=i d$, and thus

$$
p^{* *}\left(E^{\prime}\right)=p^{* *}(E)-p^{* *}(\tau p)^{* *}(E)+p^{* *} \tau^{* *}\left(E_{0}\right)=E_{0} .
$$


To generalize Corollary 23 and Corollary 24, we also need to introduce the following class of Banach algebras.

Definition 26. Let $A$ be a Banach algebra of type $(L M)$. A is said to be of type $\left(L M^{+}\right)$ if the family $\left\{\left(A_{i}, p_{i}\right)\right\}_{i \in \Lambda}$ in Theorem 4 and the subalgebra $B$ of $A$ in Theorem 5 satisfy the following extra conditions:

Each $A_{i}$ is a two-sided ideal in $A$ with a sequential BAI, each $p_{i}$ is an $A$-bimodule projection from $A$ onto $A_{i}$, and any BAI of $B$ is a BAI of $A$.

Similarly, Banach algebras of type $\left(R M^{+}\right)$and of type $\left(M^{+}\right)$can be defined.

From the proof of Proposition 8, Theorem 10, and Theorem 11, we see that all Beurling algebras $L_{1}(G, \omega)(\omega \geq 1)$ and all Fourier algebras $A(G)$ with $G$ amenable are of type $\left(M^{+}\right)$.

Proposition 27. Let $A$ be a Banach algebra of type $\left(L M^{+}\right)$(resp. of type $\left(R M^{+}\right)$) and $\mu \in \operatorname{LM}(A)$ (resp. $\mu \in R M(A)$ ). Then

$$
[\mu \in A] \text { if and only if }\left[\mu^{* *}\left(E_{1}\right)=\mu^{* *}\left(E_{2}\right) \text { for all } E_{1}, E_{2} \in \mathcal{E}\right] .
$$

Proof. We only prove the case $\mu \in L M(A)$. The case $\mu \in R M(A)$ can be proved in a similar way.

Assume $\mu^{* *}\left(E_{1}\right)=\mu^{* *}\left(E_{2}\right)$ for all $E_{1}, E_{2} \in \mathcal{E}$.

Let $B$ be the subalgebra associated with $\mu$ as in Definition 26 (cf. Theorem 5). Then any mixed identity of $B^{* *}$ is a mixed identity of $A^{* *}$, where $B^{* *}$ is identified with a subalgebra of $A^{* *}$. So, we may assume that $B=A$. Let $\left\{\left(A_{i}, p_{i}\right)\right\}_{i \in \Lambda}$ be the same family as in Definition 26 (cf. Theorem 4).

Fix an $E \in \mathcal{E}$. Let $i \in \Lambda$ and $\mu_{i}=\left.\mu\right|_{A_{i}} \in L M\left(A_{i}\right)$. To show $\mu_{i} \in A_{I}$, let $E_{1}^{0}$ and $E_{2}^{0}$ be any two mixed identities of $A_{i}^{* *}$. By Lemma $25, E_{1}^{\prime}$ and $E_{2}^{\prime}$ are mixed identities of $A$, where

$$
E_{k}^{\prime}=E-\left(\tau p_{i}\right)^{* *}(E)+\tau^{* *}\left(E_{k}^{0}\right)
$$

( $k=1,2)$, and $\tau: A_{i} \longrightarrow A$ is the inclusion map. By the assumption, we have $\mu^{* *}\left(\tau^{* *}\left(E_{1}^{0}\right)\right)=\mu^{* *}\left(\tau^{* *}\left(E_{2}^{0}\right)\right)$.

Note that $\mu \tau=\tau \mu_{i}$. So, $\tau^{* *}\left(\mu_{i}^{* *}\left(E_{1}^{0}\right)\right)=\tau^{* *}\left(\mu_{i}^{* *}\left(E_{2}^{0}\right)\right)$ and hence $\mu_{i}^{* *}\left(E_{1}^{0}\right)=\mu_{i}^{* *}\left(E_{2}^{0}\right)$, since $\tau^{* *}$ is injective. By Lemma 21(4) and Theorem $3, \mu_{i} \in A_{i}$. Since $i \in \Lambda$ is arbitrary and $A$ is of type $(L M)$, we have $\mu \in A$.

The following corollary of Proposition 27 and Corollary 22 shows that not only the answer to the open question $6 \mathrm{~h}$ ) in [27] is positive, but an even stronger and abstract form of the result can be established. 
Corollary 28. Let $A$ be a Banach algebra of type $\left(M^{+}\right)$and $m \in A^{* *}$. Then the following statements are equivalent:

(i) $m \in A$.

(ii) $m \cdot A \subseteq A$ and $m \in Z_{t}^{(l)}\left(A^{* *}\right)$.

(iii) $m \cdot A \subseteq A$ and $m \triangle E=m$ for all $E \in \mathcal{E}$.

(iv) $A \cdot m \subseteq A$ and $E \cdot m=m$ for all $E \in \mathcal{E}$.

(v) $A \cdot m \subseteq A$ and $m \in Z_{t}^{(r)}\left(A^{* *}\right)$.

At this point, we recall the following results of Ghahramani-Lau-Losert [10] on the measure algebra $M(G)$. In [10], $\Lambda(G)$ was used to denote the set of weak*-cluster points of all contractive BAIs of $L_{1}(G)$ in $L_{1}(G)^{* *}$, and it was shown that $\Lambda(G)=\left\{E \in \mathcal{E}_{R}\right.$ : $\|E\|=1\}$. Ghahramani, Lau and Losert proved in [10, Proposition 2.4(ii)] that, for all $\mu \in M(G)\left(\cong R M\left(L_{1}(G)\right)\right)$,

$$
\left[\mu \in L_{1}(G)\right] \text { if and only if }\left[E_{1} \cdot \mu=E_{2} \cdot \mu \text { for all } E_{1}, E_{2} \in \Lambda(G)\right]
$$

We observe that we also have

$$
\Lambda(G)=\left\{E \in \mathcal{E}_{L}:\|E\|=1\right\}=\{E \in \mathcal{E}:\|E\|=1\} .
$$

Therefore, Ghahramani, Lau and Losert actually proved a stronger form of Proposition 27 for the case $A=L_{1}(G)$.

We note that a Banach algebra may have a BAI without any contractive BAI. Therefore, we cannot expect that such a strengthened form of Proposition 27 holds for general Banach algebras.

However, Theorem 2 shows that for a locally compact quantum group $\mathbb{G}, L_{1}(\mathbb{G})$ must have a contractive BAI whenever it has a BAI. Naturally, one may ask whether the dual version of Ghahramani-Lau-Losert [10, Proposition 2.4(ii)] holds. This is equivalent to asking whether the set $\mathcal{E}$ in Corollary 23 can be replaced by its subset $\mathcal{E}_{1}=\{E \in \mathcal{E}$ : $\|E\|=1\}$. The answer is affirmative.

Theorem 29. Let $G$ be an amenable locally compact group and $\mu \in B(G)$. Then

$$
[\mu \in A(G)] \text { if and only if }\left[E_{1} \cdot \mu=E_{2} \cdot \mu \text { for all } E_{1}, E_{2} \in \mathcal{E}_{1}\right] \text {, }
$$

where $\mathcal{E}_{1}$ is the set of mixed identities in $A(G)^{* *}$ of norm 1.

Proof. Assume that $E_{1} \cdot \mu=E_{2} \cdot \mu$ for all $E_{1}, E_{2} \in \mathcal{E}_{1}$.

Let $H$ be any fixed $\sigma$-compact open subgroup of $G$ and let $\mu_{H}=\left.\mu\right|_{H} \in B(H)$. By $\mathrm{Hu}\left[18\right.$, Proposition 3.6], we only need to prove that $\mu_{H} \in A(H)$. We may assume that $H$ is non-compact. 
Choose a BAI $\left(e_{i}\right)_{i \in \Lambda}$ of $A(G)$ consisting of states on $V N(G)$. For each $i$, let $h_{i}=\left.e_{i}\right|_{H}$. Then $\left(h_{i}\right)$ is a BAI of $A(H)$ consisting of states on $V N(H)$.

Let $\mathcal{F}$ be the set of weak* ${ }^{*}$-cluster points of $\left(e_{i}\right)$ in $A(G)^{* *}$. Then $\mathcal{F} \subseteq \mathcal{E}_{1}$. By the assumption, we have

$$
E_{1} \cdot \mu=E_{2} \cdot \mu \text { for all } E_{1}, E_{2} \in \mathcal{F} \text {. }
$$

Note that $H$ is $\sigma$-compact and amenable. Let $\left(a_{n}\right)$ be a sequential BAI of $A(H)$. Then there exists a sequence $i_{1} \preceq i_{2} \preceq \cdots$ in $\Lambda$ such that for all $i \succeq i_{n}$, we have

$$
\left\|h_{i} a_{k}-a_{k}\right\| \leq n^{-1}(1 \leq k \leq n)
$$

Claim 1: $\left(h_{i_{n}}\right)$ is a sequential BAI of $A(H)$.

To see this, let $a \in A(H)$. Let $k_{0}$ be any fixed positive integer and $n \geq k_{0}$. Then

$\left\|h_{i_{n}} a-a\right\| \leq\left\|h_{i_{n}}\left(a-a_{k_{0}} a\right)\right\|+\left\|\left(h_{i_{n}} a_{k_{0}}-a_{k_{0}}\right) a\right\|+\left\|a_{k_{0}} a-a\right\| \leq 2\left\|a_{k_{0}} a-a\right\|+n^{-1}\|a\|$.

Therefore, $\left(h_{i_{n}}\right)$ is a sequential BAI of $A(H)$.

Claim 2: $\left(i_{n}\right)$ is cofinal in $\Lambda$.

Otherwise, there exists an $i_{0} \in \Lambda$ such that $i_{n} \preceq i_{0}$ for all $n$. In $(* *)$, take $i=i_{0}$ and let $n \longrightarrow \infty$. We have $h_{i_{0}} a_{k}=a_{k}$ for all $k$. Let $E$ be any weak*-cluster point of $\left(a_{k}\right)$ in $A(H)^{* *}$. Then $E=h_{i_{0}} \in A(H)$, contradicting the fact that $A(H)$ is non-unital (since $H$ is non-compact).

Let $\mathcal{B}$ be the set of all weak ${ }^{*}$-cluster points of $\left(h_{i_{n}}\right)$ in $A(H)^{* *}$.

Claim 3: $\mathcal{B} \subseteq\left\{\left.F\right|_{V N(H)}: F \in \mathcal{F}\right\}$.

Let $B \in \mathcal{B}$. Then there exists a subnet $\left(i_{n}^{\prime}\right)$ of $\left(i_{n}\right)$ such that $h_{i_{n}^{\prime}} \longrightarrow B$ in the weak*topology on $A(H)^{* *}$. By Claim 2, $\left(i_{n}^{\prime}\right)$ is also a subnet of $\Lambda$, so, we may assume that $e_{i_{n}^{\prime}} \longrightarrow F$ for some $F \in A(G)^{* *}$ in the weak*-topology on $A(G)^{* *}$. Thus, $F \in \mathcal{F}$. It is easy to see that $B=\left.F\right|_{V N(H)}$.

Finally, let $B \in \mathcal{B}$. By Claim $3, B=\left.F\right|_{V N(H)}$ for some $F \in \mathcal{F}$. It can be seen that $B \cdot \mu_{H}=\left.(F \cdot \mu)\right|_{V N(H)}$. By $(*)$, we have

$$
B_{1} \cdot \mu_{H}=B_{2} \cdot \mu_{H} \text { for all } B_{1}, B_{2} \in \mathcal{B} \text {. }
$$

Therefore, by Claim 1, Lemma 21(4), and Theorem 11, $\mu_{H} \in A(H)$.

Note that both $L_{1}(G)$ and $A(G)$ for amenable groups $G$ are Banach algebras of type $\left(M^{+}\right)$. Combining Corollary 28 with [10, Proposition 2.4(ii)] and Theorem 29, respectively, we obtain the following result on $L_{1}(G)$ and $A(G)$, which strengthens both LauÜlger [27, Theorem 5.4] and Miao [31, Theorem 3.2]. 
Corollary 30. Let $G$ be a locally compact group, and let $A=L_{1}(G)$, or $A(G)$ with $G$ amenable. Then, for all $m \in A^{* *}$,

$$
[m \in A] \text { if and only if }\left[A \cdot m \subseteq A \text { and } E \cdot m=m \text { for all } E \in \mathcal{E}_{1}\right]
$$

where $\mathcal{E}_{1}$ is the set of mixed identities in $A^{* *}$ of norm 1.

Remark 31. As mentioned earlier, Theorem 29 is the dual version of Ghahramani-LauLosert [10, Proposition 2.4(ii)]. A close inspection of the proof of Theorem 29 shows that this result by Ghahramani-Lau-Losert and Theorem 29 can actually be proved through a unified Banach algebraic approach.

Furthermore, we observe that, with the help of Theorem 2, such a proof with a quantum group flavour for the two classical quantum groups may be modified to establish locally compact quantum group versions of Theorem 29 and Corollary 30.

Let $G$ be a locally compact group and $m \in A(G)^{* *}$. It is proved by $\mathrm{Hu}[18$, Proposition $3.8]$ that $m \in A(G) \oplus U C B(\widehat{G})^{\perp}$ if and only if $\left.m\right|_{V N(H)} \in A(H) \oplus U C B(\widehat{H})^{\perp}$ for all $\sigma$-compact open subgroups $H$ of $G$. This fact is used in Miao's proof of [31, Theorem 3.2]. As pointed out in [18], one may not have $m \in A(G)$ even if $\left.m\right|_{V N(H)} \in A(H)$ for all $\sigma$-compact open subgroups $H$ of $G$. These observations together with Corollary 22 (the case when $A$ has a sequential BAI) motivate the definition below.

Definition 32. Let $A$ be a Banach algebra with a BAI. Assume that there exists a family $\left\{A_{i}\right\}_{i \in \Lambda}$ of closed ideals in $A$ with the following properties.

(I) Each $A_{i}$ is WSC with a sequential BAI.

(II) For each $i \in \Lambda$, there exists an $A$-bimodule projection $p_{i}$ from $A$ onto $A_{i}$.

(III) For any $m \in A^{* *}, m \in A \oplus\left(A A^{*}\right)^{\perp}$ (resp. $m \in A \oplus\left(A^{*} A\right)^{\perp}$ ) if for all $i$, $p_{i}^{* *}(m) \in A_{i}\left(\right.$ resp. $\left.p_{i}^{* *}(m) \in A_{i}\right)$.

Then $A$ is said to be of type $\left(L M^{\perp}\right)$ (resp. of type $\left(R M^{\perp}\right)$ ).

If $A$ is both of type $\left(L M^{\perp}\right)$ and of type $\left(R M^{\perp}\right)$, then $A$ is said to be of type $\left(M^{\perp}\right)$.

It can be seen that $A(G)$ is of type $\left(M^{\perp}\right)$ if $G$ is amenable, and so is $L_{1}(G, \omega)(\omega \geq 1)$ if $G$ is metrizable or $\sigma$-compact.

Naturally, one may wonder about the relationship between type $\left(L M^{+}\right)$and type $\left(L M^{\perp}\right)\left(\right.$ resp. $\left(R M^{+}\right)$and $\left.\left(R M^{\perp}\right)\right)$. Clearly, any WSC Banach algebra with a sequential BAI is both of type $\left(M^{+}\right)$and of type $\left(M^{\perp}\right)$. For a Banach algebra $A$ of type $\left(L M^{\perp}\right)$ (resp. $\left(R M^{\perp}\right)$ ) with the family $\left\{A_{i}\right\}$ as given in Definition 32 , if $A$ is an inductive union of $\left\{A_{i}\right\}$ in the sense of $\mathrm{Hu}$ [16], and the BAIs in $A_{i}$ are bounded uniformly in $i \in \Lambda$, then it can be seen that $A$ is of the type as in Theorem 4 , and hence $A$ is of type $\left(L M^{+}\right)$ (resp. $\left.\left(R M^{+}\right)\right)$. 
Conversely, for a Banach algebra $A$ of type $\left(L M^{+}\right)$(resp. of type $\left.\left(R M^{+}\right)\right)$with $B=A$ in Definition 26, if $A$ an inductive union of the family $\left\{A_{i}\right\}$ (as given in Theorem 4), then we can show that $A$ is of type $\left(L M^{\perp}\right)$ (resp. $\left.\left(R M^{\perp}\right)\right)$.

For a Banach algebra $A$ with a BAI, Lau-Ülger introduced in [27] a subspace $\Lambda_{1}$ of $A^{* *}$ and showed in $[27$, Proposition 5.7] that

$$
\Lambda_{1}=\left\{m \in A^{* *}: A \cdot m \subseteq A \text { and } E \cdot m=m \text { for all } E \in \mathcal{E}\right\} .
$$

With the Banach algebra $\left\langle A^{*} A\right\rangle^{*}$ involved, [27, Proposition 5.9] together with [27, Lemma 5.10] imply that $\Lambda_{1}=A$ if $A$ is WSC with a sequential BAI, which can now be deduced from Corollary 22. An equivalent form of the open question $6 \mathrm{~g}$ ) in [27] is whether we have $\Lambda_{1}=A$ if $A$ is WSC and non-unital with a BAI.

Proposition 27 shows that $\Lambda_{1}=A$ if $A$ is of type $\left(R M^{+}\right)$, in particular, it is true if $A=L_{1}(G, \omega)(\omega \geq 1)$ or $A=A(G)$ with $G$ amenable. We will see from Theorem 33 below that it is also the case if $A$ is of type $\left(R M^{\perp}\right)$.

We point out that so far, our study has not involved the Banach algebras $\left\langle A^{*} A\right\rangle^{*}$ and $\left\langle A A^{*}\right\rangle^{*}$ yet. We shall consider in [20] the intriguing interrelationships between these two Banach algebras and the topological centres $Z_{t}^{(l)}\left(A^{* *}\right)$ and $Z_{t}^{(r)}\left(A^{* *}\right)$.

For Banach algebras of type $\left(L M^{\perp}\right)$ or of type $\left(R M^{\perp}\right)$, we have the following variant of Theorem 18, which shows that for both Banach algebras of type $\left(M^{\perp}\right)$ and Banach algebras of type $\left(M^{+}\right)$, the assertion of Corollary 28 holds.

Theorem 33. Let $A$ be a Banach algebra and $m \in A^{* *}$. Consider the following statements.

(i) $m \in A$.

(ii) $m \cdot A \subseteq A$ and $m \in Z_{t}^{(l)}\left(A^{* *}\right)$.

(iii) $m \cdot A \subseteq A$ and $m \triangle E=m$ for all $E \in \mathcal{E}$.

(iv) $A \cdot m \subseteq A$ and $E \cdot m=m$ for all $E \in \mathcal{E}$.

(v) $A \cdot m \subseteq A$ and $m \in Z_{t}^{(r)}\left(A^{* *}\right)$.

Then (i) $\Longleftrightarrow$ (ii) $\Longleftrightarrow$ (iii) if $A$ is of type $\left(L M^{\perp}\right)$, and (i) $\Longleftrightarrow$ (iv) $\Longleftrightarrow$ (v) if $A$ is of type $\left(R M^{\perp}\right)$.

In particular, (i) - (v) are equivalent if $A$ is of type $\left(M^{\perp}\right)$.

Proof. Obviously, (i) $\Longrightarrow$ (ii) $\Longrightarrow$ (iii). Let $A$ be a Banach algebra of type $\left(L M^{\perp}\right)$, and $\left\{A_{i}\right\}$ the family of closed ideals in $A$ as in Definition 32 .

Assume (iii) holds. Let $i$ be a fixed index and let $m_{i}=p_{i}^{* *}(m) \in A_{i}^{* *}$.

Since $p_{i}: A \longrightarrow A_{i}$ is an $A$-bimodule projection,

$$
m_{i} \cdot a=p_{i}^{* *}(m) \cdot a=p_{i}^{* *}(m \cdot a)=p_{i}(m \cdot a) \in A_{i} \text { for all } a \in A_{i} .
$$


So, the condition " $m_{i} \cdot A_{i} \subseteq A_{i}$ " is satisfied.

Next, let $E_{i}$ be a mixed identity of $A_{i}^{* *}$. We show that $m_{i} \triangle E_{i}=m_{i}$. By Lemma 25, there exists an $E \in \mathcal{E}$ such that $p_{i}^{* *}(E)=E_{i}$. Note that for all $a, b \in A$, since $p_{i}: A \longrightarrow A_{i}$ is an $A$-bimodule projection and $A_{i}$ has a BAI, say $\left(e_{\alpha}\right)$, we have

$$
p_{i}(a b)=a p_{i}(b)=\lim _{\alpha} a e_{\alpha} p_{i}(b)=\lim _{\alpha} p_{i}\left(a e_{\alpha}\right) p_{i}(b)=\lim _{\alpha} p_{i}(a) e_{\alpha} p_{i}(b)=p_{i}(a) p_{i}(b) .
$$

That is, $p_{i}: A \longrightarrow A$ is an algebra homomorphism. Therefore, $p_{i}^{* *}:\left(A^{* *}, \triangle\right) \longrightarrow$ $\left(A_{i}^{* *}, \triangle\right)$ is also an algebra homomorphism. In particular, we get

$$
m_{i} \triangle E_{i}=p_{i}^{* *}(m) \triangle p_{i}^{* *}(E)=p_{i}^{* *}(m \triangle E)=p_{i}^{* *}(m)=m_{i} .
$$

By Corollary 22, $m_{i} \in A_{i}$. Since the index $i$ is arbitrary and $A$ is of type $\left(L M^{\perp}\right)$, by Definition 32(III), we have $m \in A \oplus\left(A A^{*}\right)^{\perp}$. Thus, $m=a+k$ for some $a \in A$ and $k \in\left(A A^{*}\right)^{\perp}$. Take an $E \in \mathcal{E}$. Then $k \triangle E=0$. Therefore,

$$
m=m \triangle E=a \triangle E=a \in A,
$$

i.e., (i) is true.

A similar argument shows [(i) $(\mathrm{iv}) \Longleftrightarrow(\mathrm{v})]$ for Banach algebras $A$ of type $\left(R M^{\perp}\right)$.

We conclude this section with the following result on topological centres, which is a direct consequence of Theorem 18 and Theorem 33.

Theorem 34. Let $A$ be a Banach algebra.

(i) Assume that $A$ is of type $(L M)$ or of type $\left(L M^{\perp}\right)$ and $m \in Z_{t}^{(l)}\left(A^{* *}\right)$. Then $m \in A$ if and only if $m \cdot A \subseteq A$. Therefore,

$$
Z_{t}^{(l)}\left(A^{* *}\right)=A \text { if and only if } Z_{t}^{(l)}\left(A^{* *}\right) \cdot A \subseteq A .
$$

In particular, $Z_{t}^{(l)}\left(A^{* *}\right)=A$ if $A$ is a left ideal in $A^{* *}$.

(ii) Assume that $A$ is of type $(R M)$ or of type $\left(R M^{\perp}\right)$ and $m \in Z_{t}^{(r)}\left(A^{* *}\right)$. Then $m \in A$ if and only if $A \cdot m \subseteq A$. Therefore,

$$
Z_{t}^{(r)}\left(A^{* *}\right)=A \text { if and only if } A \cdot Z_{t}^{(r)}\left(A^{* *}\right) \subseteq A .
$$

In particular, $Z_{t}^{(r)}\left(A^{* *}\right)=A$ if $A$ is a right ideal in $A^{* *}$.

Remark 35. Theorem 34(i) for $A$ being WSC with a sequential BAI was proved by Lau-Ülger [27, Theorem 3.4a)], which was also recorded in [1, Theorem 2.1(i)] and [4, Theorem 5.13], respectively. On this occasion, we would like to point out that the inclusion " $A Z_{1} \subseteq A$ " in $\left[27\right.$, Theorem 3.4] should be read as " $Z_{1} A \subseteq A$ ". 
Related to Theorem 34(i), Baker, Lau and Pym proved in [1, Theorem 2.1(iii)] that if $A$ is WSC with a BAI and $A$ is a right ideal in $A^{* *}$ satisfying $Z_{t}^{(l)}\left(A^{* *}\right) \cdot A \subseteq A$, then $Z_{t}^{(l)}\left(A^{* *}\right)=A$. Our Theorem 34 explains the remark made by Baker-Lau-Pym [1, p.196] that it seems very hard to show $Z_{t}^{(l)}\left(A^{* *}\right) \cdot A \subseteq A$ without showing that $A$ is left strongly Arens irregular (i.e., $Z_{t}^{(l)}\left(A^{* *}\right)=A$ ) - since by our result both assertions are equivalent for a large class of Banach algebras (including the ones mostly studied in the paper by Baker-Lau-Pym).

We would also like to mention the following results by Ülger. In [47], without requiring the existence of a BAI, Ülger considered commutative semisimple WSC Banach algebras $A$ which are completely continuous (i.e., for each $a \in A$, the map $A \longrightarrow A, b \rightarrow a b$, is compact). For such a Banach algebra $A$ and $m \in A^{* *}$, Ülger proved in [47, Theorem 2.2] that $m \in Z\left(A^{* *}\right)$ if and only if $\left[m \cdot A^{* *} \subseteq A\right.$ and $\left.A^{* *} \cdot m \subseteq A\right]$. In particular, if $A$ has a BAI in this case, then $Z\left(A^{* *}\right)=A$ (cf. [47, Corollary 2.2]).

\section{Applications to Losert's WORK on $A(S U(3))^{* *}$}

At the 2004 Istanbul International Conference on Abstract Harmonic Analysis, Viktor Losert showed that $Z\left(A(S U(3))^{* *}\right) \neq A(S U(3))$ in his presentation "On the centre of the bidual of Fourier algebras (the compact case)". In the current section, we discuss some consequences of this counterexample by Losert and some of our results obtained in previous sections.

First, we have the following comparison between the class of Banach algebras of type $(M)$ and the class of Neufang's type $(M F)$ Banach algebras. We call a Banach algebra $A$ to be of type $(M F)$ if $A$ has the Mazur property of level $\kappa$ and $A^{*}$ has the left $A^{* *}$ factorization property of level $\kappa$ for some cardinal $\kappa \geq \aleph_{0}$ (see Neufang [35] for the detailed description of this type of Banach algebras). See also Neufang [36] and Hu-Neufang [19] for discussions on the Mazur property of higher level.

It is shown by Hu-Neufang [19, Corollary 4.3(i)] that $A(G)$ always has the Mazur property of level $\chi(G) \cdot \aleph_{0}$. So, by Neufang [35, Theorem 2.3] and the above result of Losert, for $G=S U(3), V N(G)$ cannot have the left $A(G)^{* *}$ factorization property of level $\chi(G)\left(=\aleph_{0}\right)$, and hence $A(G)$ is not a Banach algebra of type $(M F)$. In this aspect, $A(G)$ behaves very differently from $L_{1}(G)$, since for all non-compact locally compact groups $G, L_{1}(G)$ is always of type $(M F)$ with $\kappa=\kappa(G)$ (cf. Neufang [34, $36])$. Therefore, we see that $L_{1}(G)$ for any locally compact group $G$ and $A(G)$ for any amenable group $G$ are in the class of Banach algebras of type $(M)$, while the $(M F)$-class does not include $A(G)$ for certain compact groups $G$. 
Recall that for any Banach algebra $A$, the Banach space $\left\langle A^{*} A\right\rangle^{*}$ is also a Banach algebra under the multiplication - induced by the left Arens product on $A^{* *}$. The topological centre of $\left\langle A^{*} A\right\rangle^{*}$ is defined as

$$
Z_{t}\left(\left\langle A^{*} A\right\rangle^{*}\right)=\left\{m \in\left\langle A^{*} A\right\rangle^{*}: \text { the map } n \longmapsto m \cdot n \text { is weak } \text {-weak }^{*} \text { continuous }\right\} \text {. }
$$

It can be seen that if $A$ has a bounded right approximate identity, then there exists a natural injective algebra homomorphism from $R M(A)$ to $Z_{t}\left(\left\langle A^{*} A\right\rangle^{*}\right)$. It is known that if $A=L_{1}(G)$ for any locally compact group $G$, then the embedding $R M(A) \hookrightarrow Z_{t}\left(\left\langle A^{*} A\right\rangle^{*}\right)$ is onto. See Dales-Lau [5], Hu-Neufang-Ruan [20], and Lau-Ülger [27] for all of the above discussions.

Next, we show that $S U(3)$ helps to answer Lau-Ülger [27, question 6f)] in the negative even for the commutative case. Question 6f) in [27] asked whether the embedding $R M(A) \hookrightarrow Z_{t}\left(\left\langle A^{*} A\right\rangle^{*}\right)$ is always onto if $A$ is a non-unital WSC Banach algebra with a BAI.

Proposition 36. For $G=S U(3) \times \mathbb{Z}$, we have $Z_{t}\left(U C B(\widehat{G})^{*}\right) \neq B(G)$.

Proof. Clearly, $G$ is a non-compact amenable locally compact group, and $S U(3)$ is an open subgroup of $G$. Since $Z\left(A(S U(3))^{* *}\right) \neq A(S U(3))$, by Hu-Neufang [19, Remark 8.4], $\left.Z(A(G))^{* *}\right) \neq A(G)$. Therefore, by Lau-Losert [26, Theorem 6.4], $Z_{t}\left(U C B(\widehat{G})^{*}\right) \neq$ $B(G)$.

Let $A$ be a WSC Banach algebra with a BAI, $m \in Z_{t}^{(l)}\left(A^{* *}\right)$, and $\tau_{m}: A^{*} \longrightarrow A^{*}$ the map defined by $\tau_{m}(f)=f \triangle m\left(f \in A^{*}\right)$. Another open question asked by Lau-Ülger [27] is whether the sets $\operatorname{ker}\left(\tau_{m}\right)$ and $\tau_{m}\left(\operatorname{Ball}\left(A^{*}\right)\right)$ are weak ${ }^{*}$ closed in $A^{*}$, in particular, whether it is the case when $A^{*}$ is a von Neumann algebra (cf. [27, question 6i)]). We show that the answer to this question is also negative even in the von Neumann algebra case.

For the convenience of the reader, we recall here the definition of property $(X)$, which is needed in Proposition 37 and Corollary 38 below. A Banach space $E$ is said to have property $(X)$ if the following normality criterion is satisfied: If $m$ is a functional in $E^{* *}$ such that, for every weakly unconditionally Cauchy series $\sum f_{n}$ in $E^{*}$, the equality

$$
\left\langle m, \mathrm{w}^{*}-\sum f_{n}\right\rangle=\sum\left\langle m, f_{n}\right\rangle
$$

holds, then $m \in E$, where the limit $\mathrm{w}^{*}-\sum f_{n}$ is taken in the $\sigma\left(E^{*}, E\right)$-topology (cf. Neufang [36]). 
Proposition 37. Let $A$ be a Banach algebra of type $(L M)$ or of type $\left(L M^{\perp}\right)$ with property $(X)$. Then for any $m \in Z_{t}^{(l)}\left(A^{* *}\right) \backslash A$, either $\operatorname{ker}\left(\tau_{m}\right)$ or $\tau_{m}\left(\operatorname{Ball}\left(A^{*}\right)\right)$ is not weak* closed in $A^{*}$.

Proof. Since $A$ has property $(X), A$ possesses property $(*)$ as introduced by Godefroy (see [12, p.155] and [36, Remark 2.19]). In this case, the statement "both $\operatorname{ker}\left(\tau_{m}\right)$ and $\tau_{m}\left(\operatorname{Ball}\left(A^{*}\right)\right)$ are weak* closed in $A^{*}$ " is equivalent to the statement "the map $\tau_{m}: A^{*} \longrightarrow A^{*}$ is weak*-weak* continuous" (cf. Theorem VII.8 in [12, p.172]).

Let $m \in Z_{t}^{(l)}\left(A^{* *}\right) \backslash A$. By Theorem 34(i), $m \cdot A \nsubseteq A$, i.e., $\tau_{m}^{*}(A) \nsubseteq A$. Therefore, the map $\tau_{m}: A^{*} \longrightarrow A^{*}$ is not weak*-weak* continuous, and hence either $\operatorname{ker}\left(\tau_{m}\right)$ or $\tau_{m}\left(\operatorname{Ball}\left(A^{*}\right)\right)$ is not weak* closed in $A^{*}$.

We shall see that $A(S U(3))$ is a natural counterexample to [27, question $6 \mathrm{i})$ ].

Corollary 38. Let $A$ be a separable Banach algebra with a BAI which is the predual of a von Neumann algebra. If $m \in Z_{t}^{(l)}\left(A^{* *}\right) \backslash A$, then either $\operatorname{ker}\left(\tau_{m}\right)$ or $\tau_{m}\left(\operatorname{Ball}\left(A^{*}\right)\right)$ is not weak* closed in $A^{*}$.

In particular, there exists an $m \in Z\left(A(S U(3))^{* *}\right)$ such that either $\operatorname{ker}\left(\tau_{m}\right)$ or $\tau_{m}(\operatorname{Ball}(V N(G)))$ is not weak* closed in $V N(G)$.

Proof. Obviously, $A$ is a WSC Banach algebra with a sequential BAI, and hence $A$ is of type $(M)$ (and also of type $\left(M^{\perp}\right)$ ). So, by Proposition 37, we only need to show that $A$ has property $(X)$. In fact, as the separable predual of a von Neumann algebra $M, A$ does have property $(X)$, since $M$ is countably decomposable (cf. Neufang [36, Theorem $2.18])$.

Now consider $A=A(S U(3))$. Since $S U(3)$ is metrizable and compact, $A(S U(3))$ is separable (cf. Hu [17]). Consequently, the second assertion follows from Losert's result: $Z\left(A(S U(3))^{*}\right) \neq A(G)$.

As we pointed out before, part of the present research has been stimulated by the paper [27] of Lau-Ülger, which closes with a list of 11 open problems. Indeed, most of these have now been answered, and we shall give below a brief account of the state-ofthe-art regarding their solutions.

- Questions a) and b), which are concerned with factorization of certain classes of Banach algebras, are still open. We should remark that question a) is of a fairly general nature, asking about structural properties of such classes of algebras.

- Question c) should be read as follows: "Is there a non unital, Arens regular, weakly sequentially complete Banach algebra with a BAI?" As shown by Ülger [46], the answer to this question is negative, as was conjectured by the authors of [27]. 
- Questions d), e), and j) have been answered - in the negative - in the paper [11] by Ghahramani-McClure-Meng.

- Questions f), h) and i) are answered in the present paper. Combining some of our results with Losert's work on the centre of the bidual of the Fourier algebra, we show in Proposition 36 and Corollary 38, respectively, that both questions f) and i) have negative answers in general. However, it was shown in [35] that the answer to question $\mathrm{f}$ ) is positive for Banach algebras of type $(M F)$ (as studied in [35]) which have a right approximate identity bounded by 1 .

The answer to question $\mathrm{h}$ ) was shown to be positive by Miao [31, Theorem 3.2]. This also follows from our Corollary 22 (see Corollary 24). As pointed out in Remark 20, we even obtain a generalization and a stronger form of this conjecture made by Lau-Ülger to large classes of Banach algebras (see Theorem 18 and Corollary 28, respectively). Moreover, both Lau-Ülger's result and Miao's result are further strengthened in the current paper, see our Corollary 30.

- We give a partial answer to question g) in Proposition 27 and Theorem 33 by establishing the conjecture made by Lau-Ülger for all Banach algebras $A$ of type $\left(R M^{+}\right)$ and $\left(R M^{\perp}\right)$, respectively, where $A$ is, according to the problem, assumed to have a BAI and be non unital and weakly sequentially complete.

- Finally, question $\mathrm{k}$ ) is answered in the negative in the forthcoming paper [20].

\section{REFERENCES}

1. J. Baker, A. T.-M. Lau, and J. Pym, Module homomorphisms and topological centres associated with weakly sequentially complete Banach alegbras, J. Funct. Anal. 158 (1998), 186-208.

2. E. Bédos and L. Tuset, Amenability and co-amenability for locally compact quantum groups, Internat. J. Math. 14 (2003), 865-884.

3. H. G. Dales, Banach Algebras and Automatic Continuity, London Math. Society Monographs, New Series 24, Oxford University Press, New York, 2000.

4. H. G. Dales and A. T.-M. Lau, The second duals of Beurling algebras, Mem. Amer. Math. Soc. 177 (2005), no. 836 .

5. H. A. M. Dzinotyiweyi and A. C. M. van Rooij, A characterization of the group algebra of a noncompact locally compact topological group, Quart. J. Math. Oxford Ser. (2) 41 (1990), 15-20.

6. J. De Cannière and U. Haagerup, Multipliers of the Fourier algebras of some simple Lie groups and their discrete subgroups, Amer. J. Math. 107 (1985), 455-500.

7. E. G. Effros and Z.-J. Ruan, Operator Spaces, London Math. Society Monographs, New Series 23, Oxford University Press, New York, 2000.

8. M. Enock and J.-M. Schwartz, Kac Algebras and Duality of Locally Compact Groups, SpringerVerlag, New York, 1992.

9. B. E. Forrest, V. Runde, and N. Spronk, Operator amenability of the Fourier algebra in the cbmultiplier norm, Canad. J. Math., to appear. 
10. F. Ghahramani, A. T.-M. Lau and V. Losert, Isometric isomorphisms between Banach algebras related to locally compact groups, Trans. Amer. Math. Soc. 321 (1990), 273-283.

11. F. Ghahramani, J. P. McClure, and M. Meng, On asymmetry of topological centres of the second duals of Banach algebras, Proc. Amer. Math. Soc. 126 (1998), 1765 - 1768.

12. G. Godefroy, Existence and uniqueness of isometric preduals: a survey, Contemporary Math. $\mathbf{8 5}$ (1989), 131-193.

13. E. E. Granirer, Weakly almost periodic and uniformly continuous functionals on the Fourier algebra of any locally compact group, Trans. Amer. Math. Soc. 189 (1974), 371-382.

14. E. Hewitt and K. A. Ross, Abstract Harmonic Analysis II, Springer-Verlag, New York, 1970.

15. E. Hewitt and K. A. Ross, Abstract Harmonic Analysis I, Springer-Verlag, New York, 1979.

16. Z. Hu, Inductive extreme non-Arens regularity of the Fourier algebra $A(G)$, Studia Math. 151 (2002), 247-264.

17. Z. $\mathrm{Hu}$, Maximally decomposable von Neumann algebras on locally compact groups and duality, Houston J. Math. 31 (2005), 857-881.

18. Z. Hu, Open subgroups and the centre problem for the Fourier algebra, Proc. Amer. Math. Soc. 134 (2006), 3085-3095.

19. Z. Hu and M. Neufang, Decomposability of von Neumann algebras and the Mazur property of higher level, Canad. J. Math. 58 (2006), 768-795.

20. Z. Hu, M. Neufang, and Z.-J. Ruan, On topological centre problems and their interrelationships, in preparation.

21. M. Junge, M. Neufang, and Z.-J. Ruan, A representation for locally compact quantum groups, preprint.

22. J. Kraus and Z.-J. Ruan, Multipliers of Kac algebras, International J. Math. 8 (1996), $213-248$.

23. J. Kustermans, Locally compact quantum groups in the universal setting, International J. Math. 12 (2001), 289-338.

24. J. Kustermans and S. Vaes, Locally compact quantum groups in the von Neumann algebraic setting, Math. Scand. 92 (2003), 68-92.

25. A. T.-M. Lau, Uniformly continuous functionals on the Fourier algebra of any locally compact group, Trans. Amer. Math. Soc. 251 (1979), 39-59.

26. A. T.-M. Lau and V. Losert, The $C^{*}$-algebra generated by operators with compact support on a locally compact group, J. Funct. Anal. 112 (1993), 1-30.

27. A. T.-M. Lau and A. Ülger, Topological centers of certain dual algebras, Trans. Amer. Math. Soc. 348 (1996), 1191-1212.

28. H. Leptin, Sur l'algèbre de Fourier d'un groupe localement compact, C. R. Acad. Sci. Paris, Sér. A 266 (1968), 1180-1182.

29. V. Losert, Properties of the Fourier algebra that are equivalent to amenability, Proc. Amer. Math. Soc. 92 (1984), 347-354.

30. V. Losert, Fourier-Algebra und mittelbare Gruppen, Lectures at Heidelberg University, 1985.

31. T. Miao, Unit elements in the double dual of a subalgebra of the Fourier algebra $A(G)$, preprint.

32. M. Neufang, Abstrakte Harmonische Analyse und Modulhomomorphismen über von NeumannAlgebren, Ph.D. thesis at University of Saarland, Saarbrücken, Germany, 2000.

33. M. Neufang, A unified approach to the topological centre problem for certain Banach algebras arising in abstract harmonic analysis, Arch. Math. (Basel) 82 (2004), 164-171. 
34. M. Neufang, Solution to a conjecture by Hofmeier-Wittstock, J. Funct. Anal. 217 (2004), 171-180.

35. M. Neufang, On a conjecture by Ghahramani-Lau and related problems concerning topological centres, J. Funct. Anal. 224 (2005), 217-229.

36. M. Neufang, On Mazur's property and property (X), J. Operator Theory, to appear.

37. M. Neufang, Z.-J. Ruan, and N. Spronk, Completely isometric representations of $M_{c b} A(G)$ and $U C B(\widehat{G})^{*}$, Trans. Amer. Math. Soc., to appear.

38. T. W. Palmer, Banach Algebras and General Theory of *-Algebras, Volume 1, Cambridge University Press, Cambridge, 1994.

39. V. Paulsen, Completely Bounded Maps and Operator Algebras, Cambridge Studies in Advanced Mathematics 78, Cambridge University Press, Cambridge, 2002.

40. G. Pisier, Introduction to Operator Space Theory, London Math. Society Lecture Note Series 294, Cambridge University Press, Cambridge, 2003.

41. Z.-J. Ruan, The operator amenability of $A(G)$, Amer. J. Math. 117 (1995), 1449-1474.

42. Z.-J. Ruan, Amenability of Hopf von Neumann algebras and Kac algebras, J. Funct. Anal. 139 (1996), 466-499.

43. Z.-J. Ruan, A characterization of amenable groups, manuscript, 1996.

44. M. Takesaki, Theory of Operator Algebras I, Encyclopaedia of Mathematical Sciences 124, Operator Algebras and Non-commutative Geometry V, Springer-Verlag, Berlin, 2002.

45. M. Takesaki, Theory of Operator Algebras. II, Encyclopaedia of Mathematical Sciences 125, Operator Algebras and Non-commutative Geometry VI, Springer-Verlag, Berlin, 2003.

46. A. Ülger, Arens regularity of weakly sequentially complete Banach algebras, Proc. Amer. Math. Soc. 127 (1999), 3221-3227.

47. A. Ülger, Central elements of $A^{* *}$ for certain Banach algebras $A$ without bounded approximate identities, Glasg. Math. J. 41 (1999), 369-377.

48. A. Van Daele, Locally compact quantum groups. A von Neumann algebra approach, preprint.

Department of Mathematics and Statistics, University of Windsor, Windsor, Ontario, CANADA N9B 3P4

E-mail address: zhiguohu@uwindsor.ca

School of Mathematics and Statistics, Carleton University, Ottawa, Ontario, Canada K1S 5B6

E-mail address: mneufang@math.carleton.ca

Department of Mathematics, University of Illinois, Urbana, IL 61801, USA

E-mail address: ruan@math.uiuc.edu 